Sharif University of Technology
Scientia Iranica
Transactions E: Industrial Engineering
hCIENTIA

\title{
Determining project characteristics and critical path by a new approach based on modified NWRT method and risk assessment under an interval type-2 fuzzy environment
}

\author{
Y. Dorfeshan ${ }^{a}$, S.M. Mousavi ${ }^{\mathrm{a}}$, B. Vahdani ${ }^{\mathrm{b}}$, and A. Siadat ${ }^{\mathrm{c}, *}$ \\ a. Department of Industrial Engineering, Faculty of Engineering, Shahed University, Tehran, Iran. \\ b. Department of Industrial Engineering, Faculty of Industrial and Mechanical Engineering, Qazvin Branch, Islamic Azad \\ University, Qazvin, Iran. \\ c. Laboratoire de Conception, Fabrication Commande, Arts et Métier Paris Tech, Centre de Metz, Metz, France.
}

Received 24 December 2017; received in revised form 15 March 2018; accepted 14 May 2018

\author{
KEYWORDS \\ Project scheduling; \\ Modified Node- \\ Weighted Rooted Tree \\ (NWRT) method; \\ Risk factors; \\ Interval Type-2 Fuzzy \\ Sets (IT2FSs); \\ Project \\ characteristics; \\ Project critical path.
}

\begin{abstract}
Considering the important role of risks in real-world projects and the ability of Interval Type-2 Fuzzy Sets (IT2FSs) to tackle uncertainty, this study introduces a new approach to consider risks and the correlation among risk factors by subjective judgment of experts on the probability and impact under IT2FSs. Furthermore, a new impact function used for considering the correlation among the risk factors is extended under an IT2F environment. Moreover, a new subtraction operator is introduced for the critical path analysis. The Node-Weighted Rooted Tree (NWRT) method is modified based on the proposed new operator to avoid producing negative number for characteristics of each activity. In addition, in order to deal with the uncertainty of the projects, NWRT method is developed under the IT2FSs. Finally, to illustrate the validity and capability of the proposed method, two examples derived from the literature are solved and compared.
\end{abstract}

(C) 2019 Sharif University of Technology. All rights reserved.

\section{Introduction}

The Critical Path Method (CPM) is a project management approach to planning that determines critical and non-critical activities in order to prevent timeframe problems [1]. The longest path of a project network is critical path. The CPM is a networkbased method and is designed to facilitate evaluating a project's performance and recognizing bottlenecks in the network. In addition, it is useful in practice to

*. Corresponding author. Tel.: +982151212091 E-mail addresses: sm.mousavi@shahed.ac.ir (S.M. Mousavi); ali.siadat@ensam.eu (A. Siadat)

doi: $10.24200 /$ sci. 2018.50091 .1503 schedule complex projects [1]. In the CPM, the time of activities is considered certain and deterministic. In practice, this assumption cannot always hold with satisfactory precision. Hence, Program Evaluation And Review Technique (PERT) has been introduced by using a random variable called the beta distribution to model the activity times [2]. In this method, many simplified assumptions have been considered so that it can be intensively developed in several directions under the assumption that the probability distributions of activity times are varied, compared to the beta distribution [3,4]. In such situations, for instance, Zadeh [5] used fuzzy set theory, which does not require posterior frequency distributions and, also, can deal with vague input information including feelings and emotions using subjective judgments of Decision-Makers (DMs). 
One of the most important aspects of project management is the risk management. Risk management is known as one of the ten knowledge areas of project management. Project risk has been defined by a project management institute as "an uncertain event or condition that, if it occurs, has a positive or negative effect on a project's objectives" [6]. Project risks exist because of uncertainty. In addition, there is a probability that anything known or unknown could affect the process of achieving a project's objectives. Risk management is concerned with preparations to manage these risks [6].

Several studies have focused on project and risk management in recent years. Espinoza [7] separated the project risk from the time value of money. Yim et al. [8] analyzed the impact of project classification on project risk indicators. Golini et al. [9] investigated the impact of international development projects of nongovernmental organizations. Carvalho and Rabechini Junior [10] found the link between risk management and project success. Muriana and Vizzini [11] proposed a quantitative method to assess and mitigate project risks.

Problems associated with construction projects are complex and usually involve vast uncertainties and subjectivities. Compared to other industries, the construction industry is known as a high-risk industry due to the unique features of construction activities [1214]. The incidence of project risk may have positive or negative effects on one of the project objectives, such as time, cost, safety, quality, or sustainability [15-20]. Construction projects are susceptible to considerable risks due to the involvement of a large number of parties such as owners, designers, supervision consultants, contractors, subcontractors, suppliers, manufacturers, and governments [21]. Uncertainty, vagueness, and incomplete information are inherent parts of construction projects due to the susceptibility of real-world projects to numerous risks. In this condition, fuzzy set theory is a helpful approach to the uncertainty of projects. In recent years, several types of studies have used fuzzy set theory to deal with uncertainty [22-24]. Taylan et al. [25] presented Fuzzy Analytic Hierarchy Process (FAHP) and Fuzzy Technique for Order of Preference by Similarity to Ideal Solution (FTOPSIS) to ass the risk in construction projects. Kuchta and Ptaszyńska [26] used a fuzzy-based risk register for assessing risks in construction projects. Asan et al. [27] introduced an IT2F-prioritization approach to project risk assessment.

In comparison to an ordinary Type- 1 Fuzzy Set (T1FS), Type-2 Fuzzy Set (T2FS) has a degree of membership, which is fuzzy, whereas the ordinary fuzzy set has a crisp membership function [28]. A T2FS comes with a measure of dispersion so that inherent uncertainties can be considered better, which becomes especially useful in problems in which it is difficult to determine the deterministic membership function for a fuzzy set [29]. In fact, T2FSs are more appropriate and powerful than T1FSs and provide a suitable condition for considering subjective judgments of experts. In this paper, to address the uncertainty and vagueness of realworld projects, IT2FSs are used.

IT2FSs are very beneficial and pliable to address the uncertainties in comparison to classic fuzzy sets. The great discrepancy between T2FSs and T1FSs is membership degree, which is demonstrated by a fuzzy set in $[0,1]$ for IT2FSs, instead of a crisp number in $[0,1][30,31]$. In fact, T2FSs are three-dimensional, and their membership degrees are determined by a fuzzy set at the interval $[0,1]$ and explained by both elementary and secondary memberships to supply more degrees of freedoms to tackle uncertainty. By considering the nature of the construction projects and inherent uncertainty attached to them, IT2FSs are suitable and powerful tools to tackle project uncertainty.

In recent years, many methods have been developed under IT2FSs [32-34]. Furthermore, the IT2FSs have been applied to the project management area successfully [35-37]. Bozdag et al. [38] presented a method for risk prioritization under the IT2F environment. Mohagheghi et al. [39] analyzed the project cash flow in the construction industry based on the IT2FSs. Mohagheghi et al. [40] evaluated R\&D project and portfolio selection by using an IT2F optimization approach.

In order to tackle the uncertainty of the realproject situations, fuzzy set theory was introduced by Zadeh [41]. However, classic fuzzy set theory was criticized because of its certain membership grade. In this situation, IT2FSs were proposed by Zadeh [42]. As a matter of fact, the IT2FSs are more powerful than T1FSs in dealing with uncertainty. Considering the nature of the projects and inherent uncertainty about them, the IT2FS is a suitable and powerful tool for dealing with project uncertainty. Moreover, in the project management, owing to the nature of projects and that many activities of the project may have been done for the first time, the planning process may be open to difficulties and obstacles because of the lack of information about activities. To this end, experts' opinions and judgments on activities are used. On the other hand, linguistic variables are mostly represented by fuzzy sets [43], which makes more sense than certain numbers [41]. However, classic fuzzy set theory has crisp membership grades at the interval $[0,1]$, which consequently cannot completely support various types of uncertainty present in linguistic explanations of numerical quantities or in the subjectively explained judgment of experts $[44,45]$. In this case, IT2FSs can be applied. To date, IT2FSs have been used successfully for many project management problems $[37,40]$. 
The backward recursion, explained in traditional fuzzy CPM, produces negative times because durations are explained by means of fuzzy sets and the latest starting date of the last task is set to its earliest date; however, negative number for characteristics of the activity has no physical meaning. To overcome this problem, Shanker et al. [46] introduced an analytical method for finding Fuzzy Critical Path (FCP) in a project network. Kumar and Kaur [47] presented a methodology for fuzzy critical path analysis and introduced a new subtraction operator to avoid producing negative fuzzy number. Rao and Shanker [48] carried out fuzzy critical path analysis based on the centroid of centroids of fuzzy numbers and the new subtraction method. In all subtraction methods that have been introduced, the amount of fuzzy number has increased. In fact, the crisp value of fuzzy numbers after applying the new methods increases toward the traditional methods. In this situation, a new method is developed such that the crisp value of this method is more suitable than others.

In recent years, many researchers attempt to analyze fuzzy project network and characteristics. Yakhchali and Ghodsypour [49] proposed a method for calculating the latest starting times of activities in interval-valued networks. Yakhchali and Ghodsypour [50] also introduced an approach to compute the latest starting times and activities' criticality in project network. Zareei et al. [4] presented an approach based on the analysis of events for solving FCP problem. Sireesha and Shankar [51] focused on a methodology to report project characteristics and multiple possible FCPs in a project network and introduced a graphical figure (rooted tree) to illustrate the fuzzy project network. Sireesha and Shankar [52] applied a NodeWeighted Rooted Tree (NWRT) method based on the new graphical figure (rooted tree) to illustrate project characteristics and triangular FCPs. However, this methodology produces negative fuzzy number in backward recursion. To overcome this issue and use advantages of the new graphical figure and NWRT method, this method is extended in this paper by a new operator to avoid producing negative fuzzy number because the negative fuzzy number has no physical meaning.

Determining the critical path of a project plays an important role in the planning process of real-world projects. By considering the nature of the projects and that many activities of the project may have been done for the first time, the uncertainty of project is quite high. In order to address this uncertainty, the IT2FS is a useful tool because it is more powerful in dealing with the uncertainty and provides more degree of freedom and flexibility. In this paper, the analysis of activities' risk is carried out under the IT2F environment.
In this paper, to address the uncertainty of realworld projects, IT2FSs are provided. Furthermore, a new operator is introduced to avoid producing negative fuzzy numbers for characteristics of project activities. Then, NWRT method is modified by this operator and updated in the IT2F-environment. Moreover, risks of any activity and its correlation are considered using subjective judgments of experts. In addition, a new impact function for considering the correlation of two kinds of risks for linguistic variables is developed under the IT2F environment. The novelties of this paper are summarized as follows:

- IT2FSs are used to address the uncertainty of realworld projects properly;

- A new operator is introduced to avoid producing negative fuzzy numbers under the IT2F environment;

- Risks and their correlation with each other for any activity are determined by subjective judgments under IT2FSs;

- A new impact function for considering the correlation among risk factors is developed under the IT2F environment;

- The NWRT method is introduced based on a new operator, which is modified to avoid producing negative fuzzy number;

- The modified NWRT method is extended to the IT2F environment.

The rest of this study is organized as follows: In Section 2, the proposed operator and modified NWRT method are presented. In Section 3, two examples are solved to illustrate the validity of the proposed method. In Section 4, comparative analysis is given. In Section 5, the conclusion is expressed.

\section{Proposed methodology}

In this section, the risk assessment for each task or activity by subjective judgments of DMs is presented. Then, a new impact function for considering the correlation of risk factors is developed under IT2FSs. Moreover, the NWRT method is modified based on a new subtraction operator for avoiding the production of negative fuzzy numbers. In the presented approach, the Total Float (TF) of any tasks is specified by a new characteristic path float. The Earliest Time and Latest Time (ET and LT) of each task are determined using TF. The main advantage of NWRT among other existing approaches is its simplicity and comprehensibility for any experts with low technical training. The proposed framework is depicted in Figure 1. 


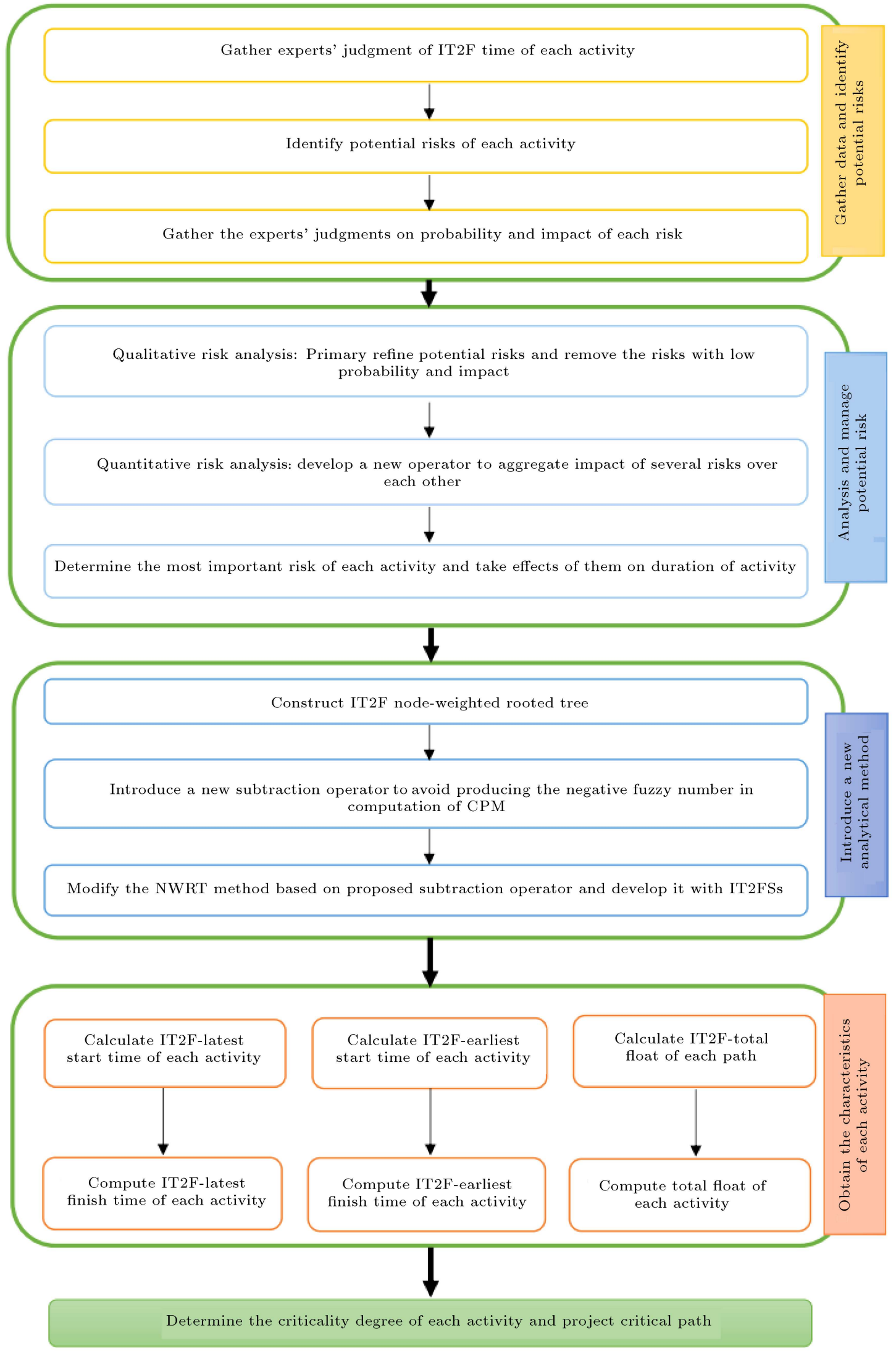

Figure 1. Graphical presentation of the proposed methodology. 


\subsection{Proposed risk assessment}

In this subsection, by considering the importance of risks in projects, risk assessment of each activity is done by the risk management group who are responsible for analyzing and determining the time of each activity. The important risks are categorized in four classes. The first class is the risk of increasing costs; the second and third ones classes are concerned with the reduction of quality and safety, respectively. Finally, the fourth class is the general risks. The experts' judgments on the probability and impact of each risk of any activities are gathered. Note that experts' judgments are expressed through linguistic variables. Then, linguistic variables are converted to the equivalent IT2F numbers. In addition, the probability of correlation among the risks is expressed by an expert, and the impact between risks is determined by a new extended method. Moreover, the most important risk of any activities is determined by their measures. Finally, the important risks' effects are converted to the time of activity. The duration of the activity is the input of the proposed NWRT method.

Procedure of the proposed methodology is provided as follows:

Step 1. Experts' judgments on the time of each activity are gathered. Furthermore, their subjective judgments on rating the probability and impact of each activity's risks are collected.

Step 2. Qualitative assessment of each risk factor is done based on experts' judgments. In other words, the risk with the very low probability and impact is removed from assessment and, then, another risk factor is considered as the input of the qualitative risk assessment stage.

Step 3. The experts' judgments on risks' probability and impact of each activity are converted to an equivalent IT2F-number by Table 1.

Step 4. A new function for obtaining the impact of two risks on each other or impact of three risks on each other, etc. is developed under IT2FSs, as adopted from Madhuri et al. [53].
If there are two fuzzy numbers $\tilde{\tilde{A}}_{1}$ and $\tilde{\tilde{A}}_{2}$ as below:

$$
\begin{aligned}
\tilde{\tilde{A}}_{1}= & \left(\tilde{A}_{1}^{U}, \tilde{A}_{1}^{L}\right) \\
= & \left(\left(a_{11}^{U}, a_{12}^{U}, a_{13}^{U}, a_{14}^{U} ; H_{1}\left(\tilde{A}_{1}^{U}\right), H_{2}\left(\tilde{A}_{1}^{U}\right)\right),\right. \\
& \left.\left(a_{11}^{L}, a_{12}^{L}, a_{13}^{L}, a_{14}^{L} ; H_{1}\left(\tilde{A}_{1}^{L}\right), H_{2}\left(\tilde{A}_{1}^{L}\right)\right)\right), \\
\tilde{\tilde{A}}_{2}= & \left(\tilde{A}_{2}^{U}, \tilde{A}_{2}^{L}\right) \\
= & \left(\left(a_{21}^{U}, a_{22}^{U}, a_{23}^{U}, a_{24}^{U} ; H_{1}\left(\tilde{A}_{2}^{U}\right), H_{2}\left(\tilde{A}_{2}^{U}\right)\right),\right. \\
& \left.\left(a_{21}^{L}, a_{22}^{L}, a_{23}^{L}, a_{24}^{L} ; H_{1}\left(\tilde{A}_{2}^{L}\right), H_{2}\left(\tilde{A}_{2}^{L}\right)\right)\right),
\end{aligned}
$$

then the addition of these two numbers is calculated as follows:

$$
\begin{aligned}
\tilde{\tilde{A}}_{3}= & \left(\tilde{A}_{3}^{U}, \tilde{A}_{3}^{L}\right) \\
= & \left(\left(a_{31}^{U}, a_{32}^{U}, a_{33}^{U}, a_{34}^{U} ; H_{1}\left(\tilde{A}_{3}^{U}\right), H_{2}\left(\tilde{A}_{3}^{U}\right)\right),\right. \\
& \left.\left(a_{31}^{L}, a_{32}^{L}, a_{33}^{L}, a_{34}^{L} ; H_{1}\left(\tilde{A}_{3}^{L}\right), H_{2}\left(\tilde{A}_{3}^{L}\right)\right)\right),
\end{aligned}
$$

where:

$$
\begin{aligned}
a_{31}^{L}= & a_{11}^{L}+a_{21}^{L}-a_{11}^{L} * a_{21}^{L} \\
a_{32}^{L}= & a_{11}^{L}+a_{22}^{L} \\
& +\left[\left(a_{12}^{L}-a_{11}^{L}\right) \frac{\min \left(H_{1}\left(\tilde{A}_{1}^{L}\right), H_{1}\left(\tilde{A}_{2}^{L}\right)\right)}{H_{1}\left(\tilde{A}_{1}^{L}\right)}\right] \\
& +\left[\left(a_{22}^{L}-a_{21}^{L}\right) \frac{\min \left(H_{1}\left(\tilde{A}_{1}^{L}\right), H_{1}\left(\tilde{A}_{2}^{L}\right)\right)}{H_{1}\left(\tilde{A}_{2}^{L}\right)}\right] \\
& -a_{12}^{L} * a_{22}^{L},
\end{aligned}
$$

Table 1. Linguistic variables and their corresponding interval Type-2 fuzzy numbers.

\begin{tabular}{ll}
\hline Linguistic variables & \multicolumn{1}{c}{ Interval Type-2 fuzzy numbers } \\
\hline Very Low $(\mathrm{VL})$ & $((0,0,0,0.1 ; 1,1),(0,0,0,0.05 ; 0.9,0.9))$ \\
Low $(\mathrm{L})$ & $((0,0.1,0.1,0.3 ; 1,1),(0.05,0.1,0.1,0.2 ; 0.9,0.9))$ \\
Medium Low $(\mathrm{ML})$ & $((0.1,0.3,0.3,0.5 ; 1,1),(0.2,0.3,0.3,0.4 ; 0.9,0.9))$ \\
Medium (M) & $((0.3,0.5,0.5,0.7 ; 1,1),(0.4,0.5,0.5,0.6 ; 0.9,0.9))$ \\
Medium High (MH) & $((0.5,0.7,0.7,0.9 ; 1,1),(0.6,0.7,0.7,0.8 ; 0.9,0.9))$ \\
High $(\mathrm{H})$ & $((0.7,0.9,0.9,1 ; 1,1),(0.8,0.9,0.9,0.95 ; 0.9,0.9))$ \\
Very High $(\mathrm{VH})$ & $((0.9,1,1,1 ; 1,1),(0.95,1,1,1 ; 0.9,0.9))$ \\
\hline
\end{tabular}




$$
\begin{aligned}
a_{33}^{L}= & a_{14}^{L}+a_{24}^{L} \\
& -\left[\left(a_{14}^{L}-a_{13}^{L}\right) \frac{\min \left(H_{2}\left(\tilde{A}_{1}^{L}\right), H_{2}\left(\tilde{A}_{2}^{L}\right)\right)}{H_{2}\left(\tilde{A}_{1}^{L}\right)}\right] \\
& -\left[\left(a_{24}^{L}-a_{23}^{L}\right) \frac{\min \left(H_{2}\left(\tilde{A}_{1}^{L}\right), H_{2}\left(\tilde{A}_{2}^{L}\right)\right)}{H_{2}\left(\tilde{A}_{2}^{L}\right)}\right] \\
& -a_{13}^{L} * a_{23}^{L}, \\
a_{34}^{L}= & a_{14}^{L}+a_{24}^{L}-a_{14}^{L} * a_{24}^{L}, \\
a_{31}^{U}= & a_{11}^{U}+a_{21}^{U}-a_{11}^{U} * a_{21}^{U}, \\
a_{32}^{U}= & a_{11}^{U}+a_{22}^{U} \\
& +\left[\left(a_{12}^{U}-a_{11}^{U}\right) \frac{\min \left(H_{1}\left(\tilde{A}_{1}^{U}\right), H_{1}\left(\tilde{A}_{2}^{U}\right)\right)}{H_{1}\left(\tilde{A}_{1}^{L}\right)}\right] \\
& -\left[\left(a_{24}^{U}-a_{23}^{U}\right) \frac{\min \left(H_{2}\left(\tilde{A}_{1}^{U}\right), H_{2}\left(\tilde{A}_{2}^{U}\right)\right)}{H_{2}\left(\tilde{A}_{2}^{L}\right)}\right. \\
& +\left[\left(a_{22}^{U}-a_{21}^{U}\right) \frac{\min \left(H_{1}\left(\tilde{A}_{1}^{U}\right), H_{1}\left(\tilde{A}_{2}^{U}\right)\right)}{H_{1}\left(\tilde{A}_{2}^{L}\right)}\right. \\
a_{34}^{U}= & a_{14}^{U}+a_{24}^{U}, \quad a_{24}^{U}-a_{14}^{U} * a_{24}^{U} . \\
a_{33}^{U}= & a_{14}^{U}+a_{12}^{U} * a_{22}^{U}, \\
& {\left.\left[a_{13}^{U}\right) \frac{\min \left(H_{2}\left(\tilde{A}_{1}^{U}\right), H_{2}\left(\tilde{A}_{2}^{U}\right)\right)}{\left.H_{1}^{U}\right)}\right] } \\
& {[4} \\
&
\end{aligned}
$$

Step 5. The risk measurement is made through the multiplication of the probability and impact. Finally, the most important risk of each activity is determined and added to the time of each activity. In fact, the output of risks assessment in this step is the updated duration for activities of project network.

\subsection{Introduced NWRT method}

In the subsection, NWRT method is expressed under IT2FSs. In fact, in this new graphical figure, (NWRT), the initial and ending nodes are considered as the root and leaf, respectively. The graphical figure illustrates all paths. Each vertex demonstrates the event, and each edge illustrates the activity. The IT2F-activity times of the project are considered as corresponding edge weights in the rooted tree. The relative importance of root can be zero. The relative importance of the ending node in each path will be the length of the path. Finally, the path with maximum length will be critical path. In order to illustrate the rooted tree, an example is shown in Table 2 and Figures 2 and 3.

Path: $1-2-4-5$

$\omega(1)=\tilde{\tilde{0}}$

Relative importance of Node $2, \omega(2)=\omega(1)+\tilde{\tilde{A}}=$ $\tilde{\tilde{0}}+\tilde{\tilde{A}}=\tilde{\tilde{A}}$

Relative importance of Node $4, \omega(4)=\omega(2)+\tilde{\tilde{D}}=$ $\tilde{\tilde{A}}+\tilde{\tilde{D}}$

Relative importance of Node $5, \omega(5)=\omega(4)+\tilde{\tilde{E}}=$ $\tilde{\tilde{A}}+\tilde{\tilde{D}}+\tilde{\tilde{E}}$.

Similarly, the weights of other nodes on other paths are calculated.

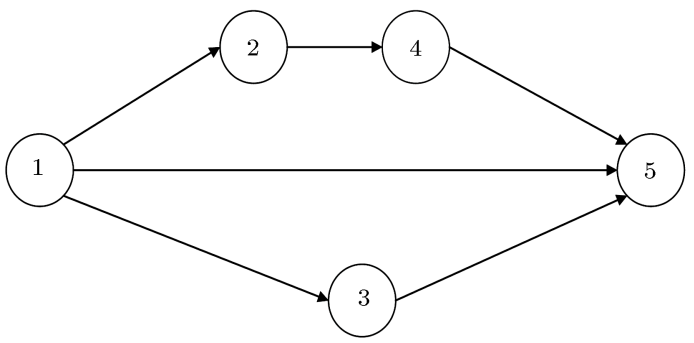

Figure 2. Fuzzy project network.

Table 2. Fuzzy activities times.

\begin{tabular}{cl}
\hline Activities & Fuzzy activities times \\
\hline $1-2$ & $\tilde{\tilde{A}}=\left(\left(a_{1}^{U}, a_{2}^{U}, a_{3}^{U}, a_{4}^{U} ; H_{1}\left(A^{U}\right), H_{2}\left(A^{U}\right)\right),\left(a_{1}^{L}, a_{2}^{L}, a_{3}^{L}, a_{4}^{L} ; H_{1}\left(A^{L}\right), H_{2}\left(A^{L}\right)\right)\right)$ \\
$1-3$ & $\tilde{\tilde{B}}=\left(\left(b_{1}^{U}, b_{2}^{U}, b_{3}^{U}, b_{4}^{U} ; H_{1}\left(B^{U}\right), H_{2}\left(B^{U}\right)\right),\left(b_{1}^{L}, b_{2}^{L}, b_{3}^{L}, b_{4}^{L} ; H_{1}\left(B^{L}\right), H_{2}\left(B^{L}\right)\right)\right)$ \\
$1-5$ & $\tilde{\tilde{C}}=\left(\left(c_{1}^{U}, c_{2}^{U}, c_{3}^{U}, c_{4}^{U} ; H_{1}\left(C^{U}\right), H_{2}\left(C^{U}\right)\right),\left(c_{1}^{L}, c_{2}^{L}, c_{3}^{L}, c_{4}^{L} ; H_{1}\left(C^{L}\right), H_{2}\left(C^{L}\right)\right)\right)$ \\
$2-4$ & $\tilde{\tilde{D}}=\left(\left(d_{1}^{U}, d_{2}^{U}, d_{3}^{U}, d_{4}^{U} ; H_{1}\left(D^{U}\right), H_{2}\left(D^{U}\right)\right),\left(d_{1}^{L}, d_{2}^{L}, d_{3}^{L}, d_{4}^{L} ; H_{1}\left(D^{L}\right), H_{2}\left(D^{L}\right)\right)\right)$ \\
$4-5$ & $\tilde{\tilde{E}}=\left(\left(e_{1}^{U}, e_{2}^{U}, e_{3}^{U}, e_{4}^{U} ; H_{1}\left(E^{U}\right), H_{2}\left(E^{U}\right)\right),\left(e_{1}^{L}, e_{2}^{L}, e_{3}^{L}, e_{4}^{L} ; H_{1}\left(E^{L}\right), H_{2}\left(E^{L}\right)\right)\right)$ \\
$3-5$ & $\tilde{\tilde{F}}=\left(\left(f_{1}^{U}, f_{2}^{U}, f_{3}^{U}, f_{4}^{U} ; H_{1}\left(F^{U}\right), H_{2}\left(F^{U}\right)\right),\left(f_{1}^{L}, f_{2}^{L}, f_{3}^{L}, f_{4}^{L} ; H_{1}\left(F^{L}\right), H_{2}\left(F^{L}\right)\right)\right)$ \\
\hline
\end{tabular}




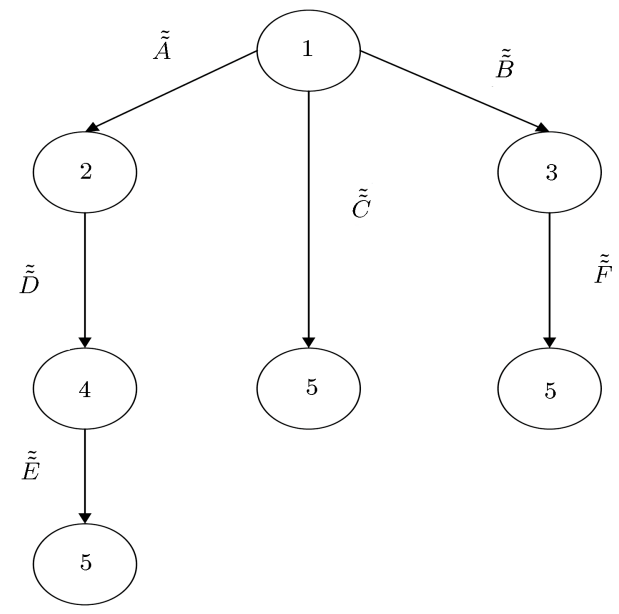

Figure 3. Rooted tree project network.

\subsection{New subtraction operation}

In this subsection, a new operator is introduced for avoiding the production of negative numbers in critical path calculations.

If there are two IT2F-numbers $\tilde{\tilde{A}}_{1}$ and $\tilde{\tilde{A}}_{2}$ based on Eq. (A.4), then a new subtraction operator $(\bullet)$ is defined as follows:

$$
\begin{aligned}
\tilde{\tilde{A}}_{1} \bullet \tilde{\tilde{A}}_{2}= & \left(\left(a_{11}^{U}-a_{21}^{U}, a_{12}^{U}-a_{22}^{U}, a_{13}^{U}-a_{23}^{U}, a_{14}^{U}-a_{24}^{U} ;\right.\right. \\
& \min \left(H_{1}\left(\tilde{A}_{1}^{U}\right), H_{1}\left(\tilde{A}_{2}^{U}\right)\right) \\
& \left.\min \left(H_{2}\left(\tilde{A}_{1}^{U}\right), H_{2}\left(\tilde{A}_{2}^{U}\right)\right)\right), \\
& \left(a_{11}^{L}-a_{21}^{L}, a_{12}^{L}-a_{22}^{L}, a_{13}^{L}-a_{23}^{L}, a_{14}^{L}-a_{24}^{L} ;\right. \\
& \min \left(H_{1}\left(\tilde{A}_{1}^{L}\right), H_{1}\left(\tilde{A}_{1}^{L}\right)\right), \\
& \left.\left.\min \left(H_{2}\left(\tilde{A}_{1}^{L}\right), H_{2}\left(\tilde{A}_{1}^{L}\right)\right)\right)\right) .
\end{aligned}
$$

However, this operator has two problems as follows:

1. Sometimes, the trapezoidal or triangular structures of fuzzy numbers are disturbed. For example, if there are two fuzzy numbers as follows:

$$
\begin{aligned}
& \tilde{\tilde{A}}_{1}=((5,7,11,12 ; 1,1),(6,8,10,11 ; 0.9,0.9)), \\
& \tilde{\tilde{A}}_{2}=((2,6,9,11 ; 1,1),(3,7,8,10 ; 0.9,0.9)),
\end{aligned}
$$

then:

$$
\begin{aligned}
\tilde{\tilde{A}}_{1}-\tilde{\tilde{A}}_{2}= & ((5-2,7-6,11-9,12-11 ; 1,1), \\
& (6-3,8-7,10-8,11-10 ; 0.9,0.9)) \\
= & ((3,1,2,1 ; 1,1),(3,1,2,1 ; 0.9,0.9)) .
\end{aligned}
$$

In this situation, the new subtraction operator is modified by using the following relation:

$$
\begin{aligned}
\tilde{\tilde{A}}_{1}-\tilde{\tilde{A}}_{2}= & \left(\left(a_{(1)}^{U}, a_{(2)}^{U}, a_{(3)}^{U}, a_{(4)}^{U} ;\right.\right. \\
& \left.\min \left(H_{1}\left(\tilde{A}_{1}^{U}\right), H_{1}\left(\tilde{A}_{2}^{U}\right)\right)\right), \\
& \left(a_{(1)}^{L}, a_{(2)}^{L}, a_{(3)}^{L}, a_{(4)}^{L} ;\right. \\
& \min \left(H_{1}\left(\tilde{A}_{1}^{L}\right), H_{1}\left(\tilde{A}_{1}^{L}\right)\right), \\
& \left.\left.\min \left(H_{2}\left(\tilde{A}_{1}^{L}\right), H_{2}\left(\tilde{A}_{1}^{L}\right)\right)\right)\right),
\end{aligned}
$$

where:

$$
a_{(1)}^{U} \leq a_{(2)}^{U} \leq a_{(3)}^{U} \leq a_{(4)}^{U},
$$

and:

$$
a_{(1)}^{L} \leq a_{(2)}^{L} \leq a_{(3)}^{L} \leq a_{(4)}^{L} .
$$

Note that $a_{(1)}^{U}$ and $a_{(1)}^{L}$ are defined as follows:

$$
\begin{aligned}
& a_{(1)}^{U}=\min \left\{a_{11}^{U}-a_{21}^{U}, a_{12}^{U}-a_{22}^{U}, a_{13}^{U}-a_{23}^{U}, a_{14}^{U}-a_{24}^{U}\right\}, \\
& a_{(1)}^{L}=\min \left\{a_{11}^{L}-a_{21}^{L}, a_{12}^{L}-a_{22}^{L}, a_{13}^{L}-a_{23}^{L}, a_{14}^{L}-a_{24}^{L}\right\} .
\end{aligned}
$$

2. If there are two fuzzy numbers as follows:

$$
\begin{aligned}
& \tilde{\tilde{A}}_{1}=((2,5,10,12 ; 1,1),(3,6,9,10 ; 0.9,0.9)), \\
& \tilde{\tilde{A}}_{2}=((3,5,8,10 ; 1,1),(4,6,7,9 ; 0.9,0.9)),
\end{aligned}
$$

then some of its elements will be negative:

$$
\begin{aligned}
\tilde{\tilde{A}}_{1}-\tilde{\tilde{A}}_{2}= & ((2-3,5-5,10-8,12-10 ; 1,1), \\
& (3-4,6-6,9-7,10-9 ; 0.9,0.9)) \\
= & ((-1,0,+2,+2 ; 1,1) \\
& (-1,0,+2,+1 ; 0.9,0.9)) .
\end{aligned}
$$

Negative numbers in the project scheduling have no physical meaning. In this condition, the introduced operator is improved as below.

At first, similar to the first problem, the structure of fuzzy numbers is modified and, then, negative numbers are removed by using the following relations:

$$
a_{(1)}^{U}=\max \left\{0, a_{11}^{U}-a_{21}^{U}\right\}
$$




$$
\begin{aligned}
a_{(2)}^{U}= & \max \left\{0,\left[\max \left\{0, a_{12}^{U}-a_{22}^{U}\right\}\right.\right. \\
& \left.\left.+\min \left\{0, a_{11}^{U}-a_{21}^{U}\right\}\right]=C^{U}\right\} \\
a_{(3)}^{U}= & \max \left\{0,\left[\max \left\{0, a_{13}^{U}-a_{23}^{U}\right\}\right.\right. \\
& \left.\left.+\min \left\{0, a_{12}^{U}-a_{22}^{U}\right\}+\min \left\{0, C^{U}\right\}\right]=G^{U}\right\} \\
a_{(4)}^{U}= & \max \left\{0,\left[\max \left\{0, a_{14}^{U}-a_{24}^{U}\right\}\right.\right. \\
& \left.\left.+\min \left\{0, a_{13}^{U}-a_{23}^{U}\right\}+\min \left\{0, G^{U}\right\}\right]\right\} \\
& \left.\left.+\min \left\{0, a_{13}^{L}-a_{23}^{L}\right\}+\min \left\{0, G^{L}\right\}\right]\right\} \\
a_{(1)}^{L}= & \max \left\{0, a_{11}^{L}-a_{21}^{L}\right\} \\
a_{(2)}^{L}= & \max \left\{0,\left[\max \left\{0, a_{12}^{L}-a_{22}^{L}\right\}\right.\right. \\
& \left.\left.+\min \left\{0, a_{11}^{L}-a_{21}^{L}\right\}\right]=C^{L}\right\} \\
a_{(4)}^{L}= & \max \left\{0,\left[\max \left\{0, a_{14}^{L}-a_{24}^{L}\right\}\right.\right. \\
a_{(3)}^{L}= & \max \left\{0,\left[\max \left\{0, a_{13}^{L}-a_{23}^{L}\right\}\right.\right. \\
& \left.\left.+\min \left\{0, a_{12}^{L}-a_{22}^{L}\right\}+\min \left\{0, C^{L}\right\}\right]=G^{L}\right\} \\
& \\
& \\
& \\
& \\
& \\
&
\end{aligned}
$$

In order to illustrate the ability of the proposed operator, the above example is solved below:

$$
\begin{aligned}
a_{(1)}^{U}= & \max \{0,2-3\}=0, \\
a_{(2)}^{U}= & \max \{0,[\max \{0,5-5\}+\min \{0,2-3\}]=-1\} \\
= & 0, \\
a_{(3)}^{U}= & \max \{0,[\max \{0,10-8\}+\min \{0,5-5\} \\
& +\min \{0,-1\}]=1\}=1, \\
a_{(4)}^{U}= & \max \{0,[\max \{0,12-10\}+\min \{0,10-8\} \\
& +\min \{0,1\}]\}=2 . \\
a_{(1)}^{L}= & \max \{0,3-4\}=0, \\
a_{(2)}^{L}= & \max \{0,[\max \{0,6-6\}+\min \{0,3-4\}]=-1\} \\
= & 0,
\end{aligned}
$$

$$
\begin{aligned}
a_{(3)}^{L}= & \max \{0,[\max \{0,9-7\}+\min \{0,6-6\} \\
& +\min \{0,-1\}]=1\}=1, \\
a_{(4)}^{L}= & \max \{0,[\max \{0,10-9\}+\min \{0,9-7\} \\
& +\min \{0,1\}]\}=1 .
\end{aligned}
$$

Generally, the new subtraction operator is defined by the following steps:

Step 1. In this step, the subtraction operator is performed as follows:

$$
\begin{aligned}
\tilde{\tilde{A}}_{1} \bullet \tilde{\tilde{A}}_{2}= & \left(\left(a_{11}^{U}-a_{21}^{U}, a_{12}^{U}-a_{22}^{U}, a_{13}^{U}-a_{23}^{U}, a_{14}^{U}-a_{24}^{U} ;\right.\right. \\
& \min \left(H_{1}\left(\tilde{A}_{1}^{U}\right), H_{1}\left(\tilde{A}_{2}^{U}\right)\right), \\
& \left.\min \left(H_{2}\left(\tilde{A}_{1}^{U}\right), H_{2}\left(\tilde{A}_{2}^{U}\right)\right)\right), \\
& \left(a_{11}^{L}-a_{21}^{L}, a_{12}^{L}-a_{22}^{L}, a_{13}^{L}-a_{23}^{L}, a_{14}^{L}-a_{24}^{L} ;\right. \\
& \min \left(H_{1}\left(\tilde{A}_{1}^{L}\right), H_{1}\left(\tilde{A}_{1}^{L}\right)\right), \\
& \left.\left.\min \left(H_{2}\left(\tilde{A}_{1}^{L}\right), H_{2}\left(\tilde{A}_{1}^{L}\right)\right)\right)\right) .
\end{aligned}
$$

If the subtraction operator does not have any of the two problems mentioned above, the operation is performed correctly. Otherwise, go to the next step.

Step 2. In this step, the subtraction operator is modified to keep the triangular and trapezoidal fuzzy structures as follows:

$$
\begin{aligned}
\tilde{\tilde{A}}_{1}-\tilde{\tilde{A}}_{2}= & \left(\left(a_{(1)}^{U}, a_{(2)}^{U}, a_{(3)}^{U}, a_{(4)}^{U} ;\right.\right. \\
& \left.\min \left(H_{1}\left(\tilde{A}_{1}^{U}\right), H_{1}\left(\tilde{A}_{2}^{U}\right)\right)\right), \\
& \left(a_{(1)}^{L}, a_{(2)}^{L}, a_{(3)}^{L}, a_{(4)}^{L} ;\right. \\
& \min \left(H_{1}\left(\tilde{A}_{1}^{L}\right), H_{1}\left(\tilde{A}_{1}^{L}\right)\right), \\
& \left.\left.\min \left(H_{2}\left(\tilde{A}_{1}^{L}\right), H_{2}\left(\tilde{A}_{1}^{L}\right)\right)\right)\right),
\end{aligned}
$$

where:

$$
a_{(1)}^{U} \leq a_{(2)}^{U} \leq a_{(3)}^{U} \leq a_{(4)}^{U}
$$

and:

$$
a_{(1)}^{L} \leq a_{(2)}^{L} \leq a_{(3)}^{L} \leq a_{(4)}^{L} .
$$


In addition, $a_{(1)}^{U}$ and $a_{(1)}^{L}$ are defined as follows:

$a_{(1)}^{U}=\min \left\{a_{11}^{U}-a_{21}^{U}, a_{12}^{U}-a_{22}^{U}, a_{13}^{U}-a_{23}^{U}, a_{14}^{U}-a_{24}^{U}\right\}$

$a_{(1)}^{L}=\min \left\{a_{11}^{L}-a_{21}^{L}, a_{12}^{L}-a_{22}^{L}, a_{13}^{L}-a_{23}^{L}, a_{14}^{L}-a_{24}^{L}\right\}$

If the results contain a negative number, go to the next step (Step 3); otherwise, the final results are obtained.

Step 3. In order to avoid producing negative numbers, unrealistic results of the proposed operator are improved as follows:

$$
\begin{aligned}
& a_{(1)}^{U}=\max \left\{0, a_{11}^{U}-a_{21}^{U}\right\}, \\
& a_{(2)}^{U}=\max \left\{0,\left[\max \left\{0, a_{12}^{U}-a_{22}^{U}\right\}\right.\right. \\
& \left.\left.+\min \left\{0, a_{11}^{U}-a_{21}^{U}\right\}\right]=C^{U}\right\}, \\
& a_{(3)}^{U}=\max \left\{0,\left[\max \left\{0, a_{13}^{U}-a_{23}^{U}\right\}\right.\right. \\
& \left.\left.+\min \left\{0, a_{12}^{U}-a_{22}^{U}\right\}+\min \left\{0, C^{U}\right\}\right]=G^{U}\right\}, \\
& a_{(4)}^{U}=\max \left\{0,\left[\max \left\{0, a_{14}^{U}-a_{24}^{U}\right\}\right.\right. \\
& \left.\left.+\min \left\{0, a_{13}^{U}-a_{23}^{U}\right\}+\min \left\{0, G^{U}\right\}\right]\right\}, \\
& a_{(1)}^{L}=\max \left\{0, a_{11}^{L}-a_{21}^{L}\right\}, \\
& a_{(2)}^{L}=\max \left\{0,\left[\max \left\{0, a_{12}^{L}-a_{22}^{L}\right\}\right.\right. \\
& \left.\left.+\min \left\{0, a_{11}^{L}-a_{21}^{L}\right\}\right]=C^{L}\right\}, \\
& a_{(3)}^{L}=\max \left\{0,\left[\max \left\{0, a_{13}^{L}-a_{23}^{L}\right\}\right.\right. \\
& \left.\left.+\min \left\{0, a_{12}^{L}-a_{22}^{L}\right\}+\min \left\{0, C^{L}\right\}\right]=G^{L}\right\}, \\
& a_{(4)}^{L}=\max \left\{0,\left[\max \left\{0, a_{14}^{L}-a_{24}^{L}\right\}\right.\right. \\
& \left.\left.+\min \left\{0, a_{13}^{L}-a_{23}^{L}\right\}+\min \left\{0, G^{L}\right\}\right]\right\} .
\end{aligned}
$$

\subsection{T2F-total float of each activity}

In this section, firstly, the TF of each path is obtained, and it is proven that $T \tilde{\tilde{F}}_{(i, j)}=\min _{s \in S_{1, n}}\left\{P \tilde{\tilde{F}}_{s} \mid(i, j) \in s\right\}$, where $T \tilde{\tilde{F}}_{(i, j)}$ represents the TF of each activity, $P \tilde{\tilde{F}}_{s}$ represents the TF of each path, and $S_{i, j}$ is a set of all paths from $i$ to $j$. The IT2F-ES time $\left(E \tilde{\tilde{S}} T_{(i, j)}\right)$ and IT2F-LS time $\left(L \tilde{\tilde{S}} T_{(i, j)}\right)$ are calculated as follows:

$$
\begin{aligned}
& E \tilde{\tilde{S}} T_{(i, j)}=\max _{s \in S_{1, i}} \tilde{\tilde{L}}_{s}=\max _{s \in S_{1, i}} \sum_{(i, j) \in s} \tilde{\tilde{t}}_{(i, j)} \\
& =\max _{s \in S_{1, i}} \sum_{(i, j) \in s}^{n}\left(\left(t_{(1, i) 1}^{U}, t_{(1, i) 2}^{U}, t_{(1, i) 3}^{U}, t_{(1, i) 4}^{U} ;\right.\right. \\
& \left.H_{1}\left(\tilde{T}_{(1, i) 1}^{U}\right), H_{2}\left(\tilde{T}_{(1, i) 1}^{U}\right)\right), \\
& \left(t_{(1, i) 1}^{L}, t_{(1, i) 2}^{L}, t_{(1, i) 3}^{L}, t_{(1, i) 4}^{L} ; H_{1}\left(\tilde{T}_{(1, i) 1}^{L}\right),\right. \\
& \left.\left.H_{2}\left(\tilde{T}_{(1, i) 1}^{L}\right)\right)\right) \\
& L \tilde{\tilde{S}} T_{(i, j)}=\max _{s \in S_{1, n}} \tilde{\tilde{L}}_{s} \bullet \max _{s \in S_{i, n}} \tilde{\tilde{L}}_{s}=\max _{s \in S_{1, n}} \sum_{(i, j) \in s} \tilde{\tilde{t}}_{(i, j)} \\
& \text { - } \max _{s \in S_{i, n}} \sum_{(i, j) \in s} \tilde{\tilde{t}}_{(i, j)}=\max _{s} \sum_{(i, j) \in s}^{n}\left(\left(t_{(1, n) 1}^{U}\right.\right. \\
& -t_{(i, n) 1}^{U}, t_{(1, n) 2}^{U}-t_{(i, n) 2}^{U}, t_{(1, n) 3}^{U} \\
& -t_{(i, n) 3}^{U}, t_{(1, n) 4}^{U}-t_{(i, n) 4}^{U} ; H_{1}\left(\tilde{T}_{1}^{U}\right) \\
& \left.H_{2}\left(\tilde{T}_{1}^{U}\right)\right),\left(t_{(1, n) 1}^{L}-t_{(i, n) 1}^{L}, t_{(1, n) 2}^{L}\right. \\
& -t_{(i, n) 2}^{L}, t_{(1, n) 3}^{L}-t_{(i, n) 3}^{L}, t_{(1, n) 4}^{L}-t_{(i, n) 4}^{L} ; \\
& \left.\left.H_{1}\left(\tilde{T}_{1}^{L}\right), H_{2}\left(\tilde{T}_{1}^{L}\right)\right)\right)=\left(\left(l s t_{(i, j)(1)}^{U},\right.\right. \\
& l s t_{(i, j)(2)}^{U}, l s t_{(i, j)(3)}^{U}, l s t_{(i, j)(4)}^{U} \text {; } \\
& \left.H_{1}\left(\tilde{T}_{(i, j)}^{U}\right), H_{2}\left(\tilde{T}_{(i, j)}^{U}\right)\right), \\
& \left(l s t_{(i, j)(1)}^{L}, l s t_{(i, j)(2)}^{L}, l s t_{(i, j)(3)}^{L}, l s t_{(i, j)(4)}^{L},\right. \\
& \left.\left.H_{1}\left(\tilde{T}_{(i, j)}^{L}\right), H_{2}\left(\tilde{T}_{(i, j)}^{L}\right)\right)\right) .
\end{aligned}
$$




$$
\begin{aligned}
P \tilde{\tilde{F}}_{S}= & \tilde{\tilde{L}}_{S}^{*} \bullet \tilde{\tilde{L}}_{S}=\max _{s \in S_{1, n}} \tilde{\tilde{L}}_{s} \bullet \tilde{\tilde{L}}_{s} \\
= & \max _{s \in S_{1, n}} \sum_{(i, j) \in s} \tilde{\tilde{t}}_{(i, j)} \bullet s \sum_{(i, j) \in s} \tilde{\tilde{t}}_{(i, j)} \\
= & \sum_{(i, j) \in s}^{n}\left(\left(\max t_{(1, n) 1}^{U}-t_{(1, n) 1}^{U}, \max t_{(1, n) 2}^{U}\right.\right. \\
& -t_{(1, n) 2}^{U}, \max t_{(1, n) 3}^{U}-t_{(1, n) 3}^{U}, \max t_{(1, n) 4}^{U} \\
& \left.-t_{(1, n) 4}^{U} ; H_{1}\left(\tilde{T}_{1}^{U}\right), H_{2}\left(\tilde{T}_{1}^{U}\right)\right),\left(\max t_{(1, n) 1}^{L}\right. \\
& -t_{(1, n) 1}^{L}, \max t_{(1, n) 2}^{L}-t_{(1, n) 2}^{L}, \max t_{(1, n) 3}^{L} \\
& -t_{(1, n) 3}^{L}, \max t_{(1, n) 4}^{L}-t_{(1, n) 4}^{L} ; H_{1}\left(\tilde{T}_{1}^{L}\right), \\
& \left.\left.H_{2}\left(\tilde{T}_{1}^{L}\right)\right)\right)=\left(\left(p f_{s(1)}^{U}, p f_{s(2)}^{U}, p f_{s(3)}^{U}, p f_{s(4)}^{U} ;\right.\right. \\
& \left.H_{1}\left(P \tilde{F}_{S}^{U}\right), H_{2}\left(P \tilde{F}_{S}^{U}\right)\right),\left(p f_{s(1)}^{L}, p f_{s(2)}^{L},\right. \\
& \left.\left.p f_{s(3)}^{L}, p f_{s(4)}^{L} ; H_{1}\left(P \tilde{F}_{S}^{L}\right), H_{2}\left(P \tilde{F}_{S}^{L}\right)\right)\right) .(18)
\end{aligned}
$$

Theorem: The IT2F-TF of each task is obtained by:

$$
T \tilde{\tilde{F}}_{(i, j)}=\min _{s \in S_{1, n}}\left\{P \tilde{\tilde{F}}_{s} \mid(i, j) \in s\right\}
$$

Proof. Consider:

$$
\begin{aligned}
& \min _{s \in S_{1, n}}\left\{P \tilde{\tilde{F}}_{s} \mid(i, j) \in s\right\} \\
& =\min \left(\max _{s \in S_{1, n}} \sum_{(i, j) \in s} \tilde{\tilde{t}}_{(i, j)} \bullet \sum_{\substack{(i, j) \in s \\
s \in S_{1, n}}} \tilde{\tilde{t}}_{(i, j)}\right) \\
& =\min \left(\max _{s \in S_{1, n}} \sum_{(i, j) \in s} \tilde{\tilde{t}}_{(i, j)} \oplus\left(\bullet \sum_{\substack{(i, j) \in s \\
s \in S_{1, n}}} \tilde{\tilde{t}}_{(i, j)}\right)\right) \\
& =\min \left(\max _{s \in S_{1, n}} \sum_{(i, j) \in s} \tilde{\tilde{t}}_{(i, j)}\right) \oplus \min \left(\bullet \sum_{\substack{(i, j) \in s \\
s \in S_{1, n}}} \tilde{\tilde{t}}_{(i, j)}\right) \\
& =\max _{s \in S_{1, n}} \sum_{(i, j) \in s} \tilde{\tilde{t}}_{(i, j)} \bullet \max \left(\sum_{\substack{(i, j) \in s \\
s \in S_{1, n}}} \tilde{\tilde{t}}_{(i, j)}\right)
\end{aligned}
$$

$$
\begin{aligned}
& =\max _{s \in S_{1, n}} \sum_{(i, j) \in s} \tilde{\tilde{t}}_{(i, j)} \bullet \max \left(\max _{s \in S_{1, i}} \sum_{(i, j) \in s} \tilde{\tilde{t}}_{(i, j)}\right. \\
& \left.\oplus \max _{s \in S_{i, n}} \sum_{(i, j) \in s} \tilde{\tilde{t}}_{(i, j)}\right) \quad \forall(i, j) \in S, \\
& =\max _{s \in S_{1, n}} \sum_{(i, j) \in s} \tilde{\tilde{t}}_{(i, j)} \bullet \max _{s \in S_{1, i}}\left(\sum_{(i, j) \in s} \tilde{\tilde{t}}_{(i, j)}\right) \\
& \bullet \max _{s \in S_{i, n}}\left(\sum_{(i, j) \in s} \tilde{\tilde{t}}_{(i, j)}\right) \quad \forall(i, j) \in S, \\
& =T \tilde{\tilde{F}}_{(i, j)} \forall(i, j) \in S .
\end{aligned}
$$

In conclusion, the IT2F-TF of each activity is given as follows:

1. At first, the IT2F-TF of each path is determined by Eq. (18);

2. Then, The IT2F-TF of each task is reported by means of the above theorem and Eq. (19).

\subsection{IT2F-earliest start and finish time}

If $\tilde{\tilde{E}}_{i}$ denotes the IT2F-earliest start occurrence of node $i$, then $\tilde{\tilde{E}}_{i}$ can be calculated as follows:

$$
\tilde{\tilde{E}}_{i}=E \tilde{\tilde{S}} T_{(i, j)}=\max \left\{w_{n, q}(i) \mid n=1,2, \cdots, m\right\},
$$

where $n$ represents the level in the rooted tree, $q$ denotes the position of node $i$ at the $n$th level from left to right, and $w_{n, q}(i)$ stands for the weight of node $i$ introduced at various levels.

In addition, the fuzzy Earliest Finish (EF) time $\left(E \tilde{\tilde{F}} T_{(i, j)}\right)$ for each activity is computed as below:

$$
\begin{aligned}
E \tilde{\tilde{F}} T_{(i, j)}= & \tilde{\tilde{E}}_{i}+\tilde{\tilde{t}}_{(i, j)}=\max \left\{w_{k, q}(i) \mid k=1,2, \cdots, m\right\} \\
& +\tilde{\tilde{t}}_{(i, j)} .
\end{aligned}
$$

Note that the computation of IT2F-Earliest Start (ES) time by the proposed method is easier than by the conventional approach, and it will be straightly gained from the rooted tree. However, the calculation of EF time for each activity is exactly similar to that by the conventional method.

\subsection{IT2F-latest start and finish time}

In this subsection, with respect to IT2F-TF of each task, the IT2F-LS time $\left(L \tilde{\tilde{S}} T_{(i, j)}\right)$ can be calculated by: 


$$
\begin{aligned}
L \tilde{\tilde{S}} T_{(i, j)}= & T \tilde{\tilde{F}}_{(i, j)} \oplus E \tilde{\tilde{S}}_{(i, j)}=\left(\left(t f_{(i, j) 1}^{U}\right.\right. \\
& +e s t_{(i, j) 1}^{U}, t f_{(i, j) 2}^{U}+e s t_{(i, j) 2}^{U}, t f_{(i, j) 3}^{U} \\
& +e s t_{(i, j) 3}^{U}, t f_{(i, j) 4}^{U}+e s t_{(i, j) 4}^{U} ; \\
& \min \left(H_{1}\left(T \tilde{F}^{U}\right), H_{1}\left(E \tilde{\tilde{S}} T^{U}\right)\right) \\
& \left.\min \left(H_{2}\left(T \tilde{F}^{U}\right), H_{2}\left(E \tilde{\tilde{S}} T^{U}\right)\right)\right) \\
& \left(t f_{(i, j) 1}^{L}+e s t_{(i, j) 1}^{L}, t f_{(i, j) 2}^{L}+e s t_{(i, j) 2}^{L},\right. \\
& t f_{(i, j) 3}^{L}+e s t_{(i, j) 3}^{L}, t f_{(i, j) 4}^{L}+e s t_{(i, j) 4}^{L} \\
& \min \left(H_{1}\left(T \tilde{F}^{L}\right), H_{1}\left(E \tilde{\tilde{S}} T^{L}\right)\right) \\
& \left.\min \left(H_{2}\left(T \tilde{F}^{L}\right), H_{2}\left(E \tilde{\tilde{S}} T^{L}\right)\right)\right) .
\end{aligned}
$$

Furthermore, the IT2F-LF time of each task can be obtained by using the IT2F-LS time as follows:

$$
\begin{aligned}
\tilde{\tilde{L}}_{j}= & L \tilde{\tilde{F}} T_{(i, j)}=L \tilde{\tilde{S}} T_{(i, j)} \oplus \tilde{\tilde{t}}_{(i, j)} \\
= & \left(\left(l s t_{(i, j) 1}^{U}+t_{(i, j) 1}^{U}, l s t_{(i, j) 2}^{U}+t_{(i, j) 2}^{U}, l s t_{(i, j) 3}^{U}\right.\right. \\
& +t_{(i, j) 3}^{U}, l s t_{(i, j) 4}^{U}+t_{(i, j) 4}^{U} ; \\
& \min \left(H_{1}\left(L \tilde{S} T^{U}\right), H_{1}\left(\tilde{T}^{U}\right)\right) \\
& \left.\min \left(H_{2}\left(L \tilde{S} T^{U}\right), H_{2}\left(\tilde{T}^{U}\right)\right)\right) \\
& \left(l s t_{(i, j) 1}^{L}+t_{(i, j) 1}^{L}, l s t_{(i, j) 2}^{L}+t_{(i, j) 2}^{L}, l s t_{(i, j) 3}^{L}\right. \\
& +t_{(i, j) 3}^{L}, l s t_{(i, j) 4}^{L}+t_{(i, j) 4}^{L} ; \\
& \min \left(H_{1}\left(L \tilde{S} T^{L}\right), H_{1}\left(\tilde{T}^{L}\right)\right) \\
& \left.\left.\min \left(H_{2}\left(L \tilde{S} T^{L}\right), H_{2}\left(\tilde{T}^{L}\right)\right)\right)\right)
\end{aligned}
$$

Nevertheless, the computation of IT2F-latest finish and start times is easier and simpler than that by the traditional method.

\subsection{Overview of the proposed method}

In this subsection, an overview of the proposed method is carried out to understand the new method better. In addition, the proposed framework is demonstrated in Figure 1. The main steps of the proposed method are defined below:

Step 1. Gather experts' opinions on the time of each activity;
Step 2. Identify the potential risks of each activity; Step 3. Collect experts' judgments on the probability and impact of potential risks;

Step 4. Do the primary refinement of risks and remove the risks with low probability and impacts for the qualitative risk analysis;

Step 5. Convert experts' judgments on the probability and impact of each risk to equivalent IT2Fnumbers by using Table 1 ;

Step 6. Obtain the impact of $k$ risks on each other by a new developed operator, which is explained in Eqs. (1) to (4);

Step 7. Determine the most important risk of each activity by calculating the risk measurements of each activity and considering the impact of the most important risk on the duration of activities based on Step 5 of subsection 2.1;

Step 8. Construct a rooted tree project network based on Table 2 and Figure 3;

Step 9. Introduce a new subtraction operator using Eqs. (10) to (17) to avoid producing negative numbers;

Step 10. Modify the NWRT method by the new subtraction operator:

Step 10-1. Compute the IT2F-TF of each path by Eq. (18);

Step 10-2. Calculate the IT2F-TF of each activity via Eq. (19);

Step 10-3. Compute the IT2F-ES time of each task by means of Eq. (21);

Step 10-4. Calculate the IT2F-EF time of each task by Eq. (22);

Step 10-5. Obtain the IT2F-LS time of each task by means of Eq. (23);

Step 10-6. Calculate the IT2F-LF time of each task via Eq. (24).

Step 11. Determine the criticality degree of each task and the project critical path by Eq. (A.8).

\section{Application}

In this section, to illustrate the applicability and ability of the proposed method, first, an example taken from literature has been adopted and solved [52]. The project network is shown in Figure 4. In addition, experts' judgments on the time of each activity are illustrated in Table 3. Furthermore, the important risks of each activity and the probability and impact of each one are gathered from an expert and demonstrated in Tables 4 to 10 . Generally, the probability and impact of each risk of activities are gathered from experts, and the impact of each risk on each other is determined by 


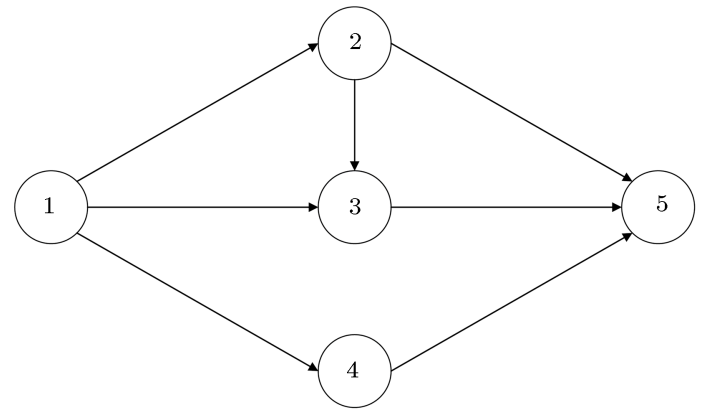

Figure 4. Project network.

Table 3. IT $2 \mathrm{~F}$-activities time.

\begin{tabular}{cl}
\hline Activities & Expert \\
\hline $1-2$ & $((10,15,20,25 ; 1,1),(12,16,19,23 ; 0.9,0.9))$ \\
$1-3$ & $((30,40,50,60 ; 1,1),(35,45,45,55 ; 0.9,0.9))$ \\
$1-4$ & $((15,23,30,37 ; 1,1),(18,25,30,35 ; 0.9,0.9))$ \\
$2-3$ & $((30,45,60,75 ; 1,1),(35,50,55,65 ; 0.9,0.9))$ \\
$2-5$ & $((60,125,180,240 ; 1,1),(90,140,160,210 ; 0.9,0.9))$ \\
$3-5$ & $((60,125,180,240 ; 1,1),(90,140,160,210 ; 0.9,0.9))$ \\
$4-5$ & $((60,125,180,240 ; 1,1),(90,140,160,210 ; 0.9,0.9))$ \\
\hline
\end{tabular}

using a new function that is developed under IT2FSs. Then, the most important risk of each activity is specified based on the risk level; finally, the impact of the most important risk is considered as the time of activities and added to the initial time of activities. Furthermore, the project network is updated by new activities' times. Moreover, the NWRT method, which was originally proposed by Sireesha and Shankar [52], is modified by means of the new subtraction method to avoid producing negative numbers in determining the characteristics of each activity.

Then, the linguistic variables are converted to equivalent IT2F-numbers, and the impacts of all risks are determined by Eqs. (11) and (12). Moreover, the risk measurement based on multiple probability and impact is calculated, and the most important risk of each activity is specified. Finally, the impact of the most important risk of each activity is regarded as the time of activity and is added to the duration of activities. The new duration of activities is depicted in Table 11. In addition, the IT2F node-weighted rooted tree is depicted in Figure 5.

For comparative purposes, the Length of Paths $(L P)$ is computed by Eq. (A.8) as follows:

$$
\begin{aligned}
& L \tilde{\tilde{P}}_{1-2-3-5}=((136.1,302.8,426,623.35 ; 1,1), \\
& (206.87,337.3,383.15,520.13 ; 0.9,0.9))=367 ; \\
& L \tilde{\tilde{P}}_{1-2-5}=((87,201.67,228,434.5 ; 1,1), \\
& (136.8,224.67,257.83,358.8 ; 0.9,0.9))=248.74 ; \\
& L \tilde{\tilde{P}}_{1-3-5}=((119.3,263.4,368.93,539.6 ; 1,1), \\
& \quad(183.53,295.3,328.7,453.07 ; 0.9,0.9))=319.05 ; \\
& L \tilde{\tilde{P}}_{1-4-5}=((88.38,221.3,314.3,482.3 ; 1,1), \\
& (145.44,246.82,284.1,399 ; 0.9,0.9))=272.79 .
\end{aligned}
$$

Table 4. Probability and impact of risks in activities 1-2.

\begin{tabular}{lcc}
\hline & \multicolumn{2}{c}{ Expert } \\
\cline { 2 - 3 } Risks & Probability & Impact \\
\hline Risk of increasing cost $=R_{1}$ & $\mathrm{ML}$ & $\mathrm{M}$ \\
Risk of reducing quality $=R_{2}$ & $\mathrm{M}$ & $\mathrm{ML}$ \\
Risk of reducing safety $=R_{3}$ & $\mathrm{MH}$ & $\mathrm{M}$ \\
Correlation between $R_{1}$ and $R_{2}$ & $\mathrm{~L}$ & Calculation via Eqs. (3) and (4) \\
Correlation between $R_{1}$ and $R_{3}$ & $\mathrm{ML}$ & Calculation via Eqs. (3) and (4) \\
Correlation between $R_{2}$ and $R_{3}$ & $\mathrm{ML}$ & Calculation via Eqs. (3) and (4) \\
Correlation among $R_{1}, R_{2}$ and $R_{3}$ & $\mathrm{~L}$ & Calculation via Eqs. (3) and (4) \\
\hline
\end{tabular}

Table 5. Probability and impact of risks in activities 1-3.

\begin{tabular}{lcc}
\hline & \multicolumn{2}{c}{ Expert } \\
\cline { 2 - 3 } Risks & Probability & Impact \\
\hline Risk of increasing cost $=R_{1}$ & $\mathrm{M}$ & $\mathrm{L}$ \\
Risk of reducing quality $=R_{2}$ & $\mathrm{ML}$ & $\mathrm{ML}$ \\
General risks $=R_{4}$ & $\mathrm{~L}$ & $\mathrm{M}$ \\
Correlation between $R_{1}$ and $R_{2}$ & $\mathrm{~L}$ & Calculation via Eqs. (3) and (4) \\
Correlation between $R_{1}$ and $R_{4}$ & $\mathrm{VL}$ & Calculation via Eqs. (3) and (4) \\
Correlation between $R_{2}$ and $R_{4}$ & $\mathrm{VL}$ & Calculation via Eqs. (3) and (4) \\
Correlation among $R_{1}, R_{2}$ and $R_{4}$ & $\mathrm{VL}$ & Calculation via Eqs. (3) and (4) \\
\hline
\end{tabular}


Table 6. Probability and impact of risks in activities 1-4.

\begin{tabular}{lcc}
\hline & \multicolumn{2}{c}{ Expert } \\
\cline { 2 - 3 } \multicolumn{1}{c}{ Risks } & Probability & Impact \\
\hline Risk of reducing quality $=R_{2}$ & $\mathrm{M}$ & $\mathrm{ML}$ \\
Risk of reducing safety $=R_{3}$ & $\mathrm{MH}$ & $\mathrm{ML}$ \\
General risks $=R_{4}$ & $\mathrm{MH}$ & $\mathrm{L}$ \\
Correlation between $R_{2}$ and $R_{3}$ & $\mathrm{M}$ & Calculation via Eqs. (3) and (4) \\
Correlation between $R_{2}$ and $R_{4}$ & $\mathrm{ML}$ & Calculation via Eqs. (3) and (4) \\
Correlation between $R_{3}$ and $R_{4}$ & $\mathrm{M}$ & Calculation via Eqs. (3) and (4) \\
Correlation among $R_{2}, R_{3}$ and $R_{4}$ & $\mathrm{ML}$ & Calculation via Eqs. (3) and (4) \\
\hline
\end{tabular}

Table 7. Probability and impact of risks in activities 2-3.

\begin{tabular}{lcc}
\hline \multicolumn{1}{c}{ Risks } & \multicolumn{2}{c}{ Expert } \\
\cline { 2 - 3 } & Probability & Impact \\
\hline Risk of increasing cost $=R_{1}$ & $\mathrm{M}$ & $\mathrm{ML}$ \\
General risks $=R_{4}$ & $\mathrm{M}$ & $\mathrm{ML}$ \\
Correlation between $R_{1}$ and $R_{4}$ & $\mathrm{ML}$ & Calculation via Eqs. (3) and (4) \\
\hline
\end{tabular}

Table 8. Probability and impact of risks in activities $2-5$.

\begin{tabular}{lcc}
\hline \multicolumn{1}{c}{ Risks } & \multicolumn{2}{c}{ Expert } \\
\cline { 2 - 3 } & Probability & Impact \\
\hline Risk of increasing cost $=R_{1}$ & $\mathrm{ML}$ & $\mathrm{M}$ \\
Risk of reducing safety $=R_{3}$ & $\mathrm{~L}$ & $\mathrm{MH}$ \\
Correlation between $R_{1}$ and $R_{3}$ & $\mathrm{VL}$ & Calculation via Eqs. (3) and (4) \\
\hline
\end{tabular}

Table 9. Probability and impact of risks in activities $3-5$.

\begin{tabular}{lcc}
\hline \multirow{2}{c}{ Risks } & \multicolumn{2}{c}{ Expert } \\
\cline { 2 - 3 } & Probability & Impact \\
\hline Risk of reducing quality $=R_{2}$ & $\mathrm{M}$ & $\mathrm{M}$ \\
Risk of reducing safety $=R_{3}$ & $\mathrm{MH}$ & $\mathrm{ML}$ \\
Correlation between $R_{2}$ and $R_{3}$ & $\mathrm{M}$ & Calculation via Eqs. (3) and (4) \\
\hline
\end{tabular}

Table 10. Probability and impact of risks in activities 4-5.

\begin{tabular}{lcc}
\hline \multicolumn{1}{c}{ Risks } & \multicolumn{2}{c}{ Expert } \\
\cline { 2 - 3 } & Probability & Impact \\
\hline Risk of increasing cost $=R_{1}$ & $\mathrm{M}$ & $\mathrm{ML}$ \\
General risks $=R_{4}$ & $\mathrm{M}$ & $\mathrm{ML}$ \\
Risk of reducing safety $=R_{3}$ & $\mathrm{ML}$ & $\mathrm{M}$ \\
Correlation between $R_{1}$ and $R_{4}$ & $\mathrm{ML}$ & Calculation via Eqs. (3) and (4) \\
Correlation between $R_{1}$ and $R_{3}$ & $\mathrm{~L}$ & Calculation via Eqs. (3) and (4) \\
Correlation between $R_{3}$ and $R_{4}$ & $\mathrm{~L}$ & Calculation via Eqs. (3) and (4) \\
Correlation among $R_{1}, R_{3}$, and $R_{4}$ & $\mathrm{~L}$ & Calculation via Eqs. (3) and (4) \\
\hline
\end{tabular}

Table 11. New durations for activities.

\begin{tabular}{cl}
\hline Activities & \multicolumn{1}{c}{ Expert } \\
\hline $1-2$ & $((13,22.5,30,42.5 ; 1,1),(16.8,24,28.5,36.8 ; 0.9,0.9))$ \\
$1-3$ & $((35,54.67,68.3,94 ; 1,1),(44.3,61.5,61.5,80.67 ; 0.9,0.9))$ \\
$1-4$ & $((16.95,32.58,42.5,62.28 ; 1,1),(23.04,35.42,42.5,54.6 ; 0.9,0.9))$ \\
$2-3$ & $((38.9,71.55,95.4,135.25 ; 1,1),(50.87,79.5,87.45,110.93 ; 0.9,0.9))$ \\
$2-5$ & $((74,179.17,258,392 ; 1,1),(120,200.67,229.3,322 ; 0.9,0.9))$ \\
$3-5$ & $((84.2,208.75,300.6,445.6 ; 1,1),(139.2,233.8,267.2,372.4 ; 0.9,0.9))$ \\
$4-5$ & $((71.4,188.75,271.8,420 ; 1,1),(122.4,211.4,241.6,344.4 ; 0.9,0.9))$ \\
\hline
\end{tabular}




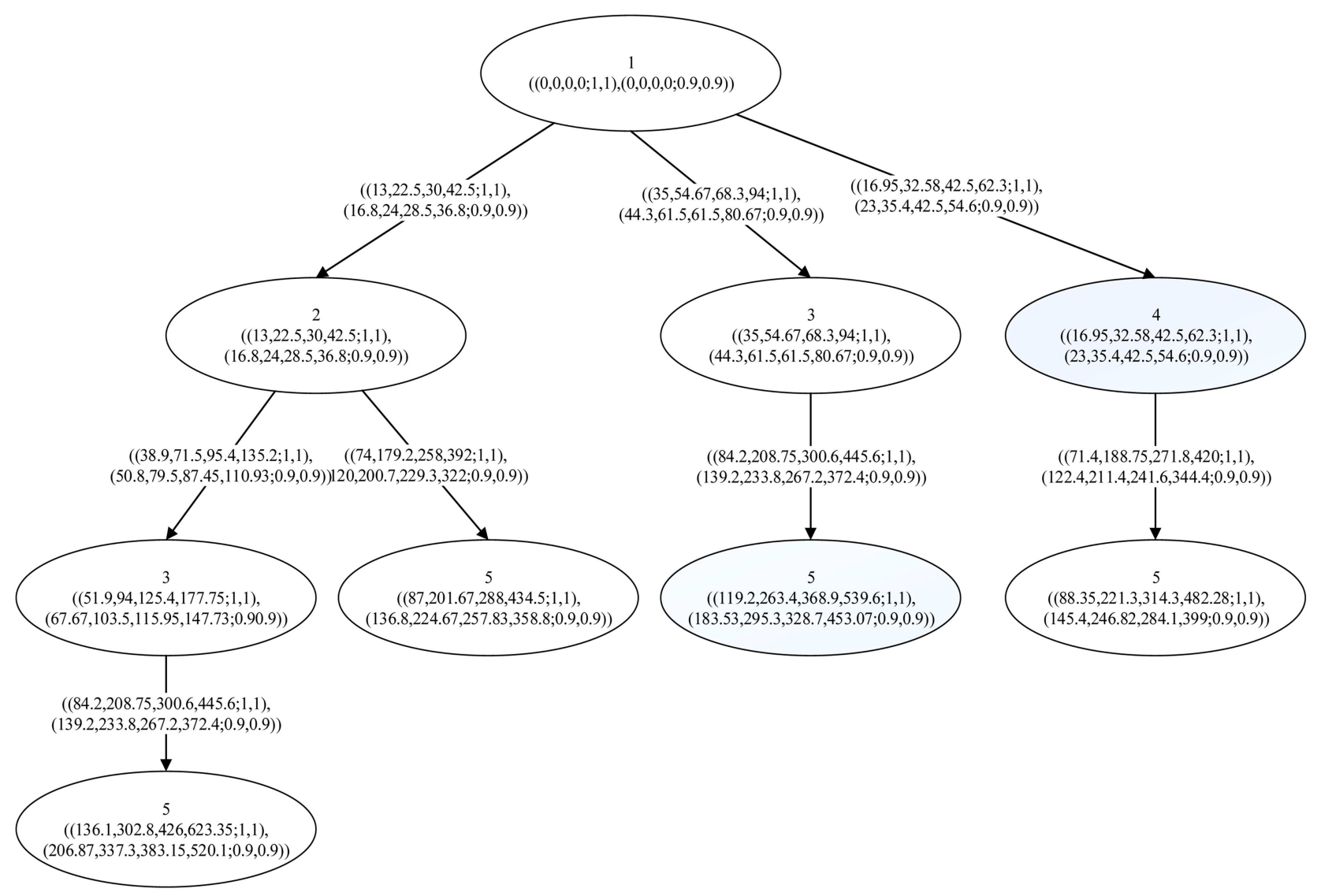

Figure 5. IT2F node-weighted rooted tree.

Table 12. Total float of each path.

\begin{tabular}{ccc}
\hline Path & Length of the path & Total float of each path \\
\hline \multirow{2}{*}{$1-2-3-5$} & $((136.1,302.8,426,623.35 ; 1,1)$, & $((0,0,0,0 ; 1,1)$, \\
& $(206.87,337.3,383.15,520.13 ; 0.9,0.9))$ & $(0,0,0,0 ; 0.9,0.9))$ \\
& $((87,201.67,228,434.5 ; 1,1)$, & $((49.1,101.13,138,188.85 ; 1,1)$, \\
$1-2-5$ & $(136.8,224.67,257.83,358.8 ; 0.9,0.9))$ & $(70.07,112.63,125.32,161.3 ; 0.9,0.9))$ \\
& & \\
$1-3-5$ & $((119.3,263.4,368.93,539.6 ; 1,1)$, & $((16.9,39.38,57.07,83.75 ; 1,1)$, \\
& $(183.53,295.3,328.7,453.07 ; 0.9,0.9))$ & $(23.3,42,54.45,67.07 ; 0.9,0.9))$ \\
& & \\
$1-4-5$ & $((88.38,221.3,314.3,482.3 ; 1,1)$, & $((47.75,81.47,111.7,141.07 ; 1,1)$, \\
& $(145.44,246.82,284.1,399 ; 0.9,0.9))$ & $(61.43,90.48,9.05,121.13 ; 0.9,0.9))$ \\
\hline
\end{tabular}

Based on the compared results, it is clear that IT2Fcritical path is 1-2-3-5. IT2F-TF of the paths is computed by means of Eq. (18) and illustrated in Table 12.

Moreover, the IT2F-TF of each activity is computed by using Eq. (19) and presented in Table 13.

For instance:

$T \tilde{\tilde{F}}_{(1,2)}=\min \left\{P \tilde{\tilde{F}}_{(1-2-3-5)}, P \tilde{\tilde{F}}_{(1-2-5)}\right\}$

$$
\begin{aligned}
= & \left.\min \left\{\begin{array}{l}
((0,0,0,0 ; 1,1),(0,0,0,0 ; 0.9,0.9)), \\
(49.1,101.1,138,188.85 ; 1,1), \\
(70.07,112.63,125.32,161.3 ; 0.9,0.9)
\end{array}\right)\right\} \\
= & ((0,0,0,0 ; 1,1),(0,0,0,0 ; 0.9,0.9)) .
\end{aligned}
$$

Furthermore, the IT2F-earliest occurrence time of each node $i$ is calculated by means of Eq. (21). 
Table 13. IT2F-total float, earliest, and latest times.

\begin{tabular}{|c|c|c|c|}
\hline Act. $^{a}$ & Activity time & Total float of each activity & Earliest start time \\
\hline $1-2$ & $\begin{array}{c}((13,22.5,30,42.5 ; 1,1) \\
(16.8,24,28.5,36.8 ; 0.9,0.9))\end{array}$ & $\begin{array}{c}((0,0,0,0 ; 1,1) \\
(0,0,0,0 ; 0.9,0.9))\end{array}$ & $\begin{array}{c}((0,0,0,0 ; 1,1) \\
(0,0,0,0 ; 0.9,0.9))\end{array}$ \\
\hline $1-3$ & $\begin{array}{c}((35,54.67,68.3,94 ; 1,1), \\
(44.3,61.5,61.5,80.67 ; 0.9,0.9))\end{array}$ & $\begin{array}{l}((16.9,39.38,57.06,83.75 ; 1,1) \\
(23.3,42,54.45,67.07 ; 0.9,0.9))\end{array}$ & $\begin{array}{c}((0,0,0,0 ; 1,1) \\
(0,0,0,0 ; 0.9,0.9))\end{array}$ \\
\hline $1-4$ & $\begin{array}{c}((16.95,32.58,42.5,62.28 ; 1,1), \\
(23.04,35.42,42.5,54.6 ; 0.9,0.9))\end{array}$ & $\begin{array}{c}((47.75,81.47,111.7,141.07 ; 1,1) \\
(61.4,90.48,99.05,121.13 ; 0.9,0.9))\end{array}$ & $\begin{array}{c}((0,0,0,0 ; 1,1) \\
(0,0,0,0 ; 0.9,0.9))\end{array}$ \\
\hline $2-3$ & $\begin{array}{c}((38.9,71.55,95.4,135.25 ; 1,1) \\
(50.87,79.5,87.45,110.93 ; 0.9,0.9))\end{array}$ & $\begin{array}{c}((0,0,0,0 ; 1,1) \\
(0,0,0,0 ; 0.9,0.9))\end{array}$ & $\begin{array}{c}((13,22.5,30,42.5 ; 1,1), \\
(16.8,24,28.5,36.8 ; 0.9,0.9))\end{array}$ \\
\hline $2-5$ & $\begin{array}{c}((74,179.17,258,392 ; 1,1) \\
(120,200.67,229.3,322 ; 0.9,0.9))\end{array}$ & $\begin{array}{c}((49.1,101.13,138,188.85 ; 1,1) \\
(70.07,112.63,125.32,161.3 ; 0.9,0.9))\end{array}$ & $\begin{array}{c}((13,22.5,30,42.5 ; 1,1) \\
(16.8,24,28.5,36.8 ; 0.9,0.9))\end{array}$ \\
\hline $3-5$ & $\begin{array}{c}((84.2,208.75,300.6,445.6 ; 1,1) \\
(139.2,233.8,267.2,372.4 ; 0.9,0.9))\end{array}$ & $\begin{array}{c}((0,0,0,0 ; 1,1) \\
(0,0,0,0 ; 0.9,0.9))\end{array}$ & $\begin{array}{c}((51.9,94.05,125.4,177.75 ; 1,1) \\
(67.67,103.5,115.95,147.73 ; 0.9,0.9))\end{array}$ \\
\hline \multirow[t]{11}{*}{$4-5$} & $\begin{array}{c}((71.4,188.75,271.8,420 ; 1,1) \\
(122.4,211.4,241.6,344.4 ; 0.9,0.9)) \\
\end{array}$ & $\begin{array}{l}((47.75,81.47,111.7,141.07 ; 1,1) \\
(61.4,90.48,99.05,121.13 ; 0.9,0.9))\end{array}$ & $\begin{array}{c}((16.95,32.58,42.5,62.28 ; 1,1) \\
(23.04,35.42,42.5,54.6 ; 0.9,0.9)) \\
\end{array}$ \\
\hline & Earliest finish time & Latest start time & Latest finish time \\
\hline & $((13,22.5,30,42.5 ; 1,1)$ & $((0,0,0,0 ; 1,1)$ & $((13,22.5,30,42.5 ; 1,1)$ \\
\hline & $(16.8,24,28.5,36.8 ; 0.9,0.9))$ & $(0,0,0,0 ; 0.9,0.9))$ & $(16.8,24,28.5,36.8 ; 0.9,0.9))$ \\
\hline & $\begin{array}{c}((35,54.67,68.3,94 ; 1,1) \\
(44.3,61.5,61.5,80.67 ; 0.9,0.9))\end{array}$ & $\begin{array}{l}((16.9,39.38,57.06,83.75 ; 1,1), \\
(23.3,42,54.45,67.07 ; 0.9,0.9))\end{array}$ & $\begin{array}{c}((51.9,94.05,125.4,177.75 ; 1,1) \\
(67.67,103.5,115.95,147.73 ; 0.9,0.9))\end{array}$ \\
\hline & $\begin{array}{c}((16.95,32.58,42.5,62.28 ; 1,1) \\
(23.04,35.42,42.5,54.6 ; 0.9,0.9))\end{array}$ & $\begin{array}{c}((47.75,81.47,111.7,141.07 ; 1,1) \\
(61.4,90.48,99.05,121.13 ; 0.9,0.9))\end{array}$ & $\begin{array}{c}((64.7,114.05,154.2,203.35 ; 1,1) \\
(84.47,125.9,141.55,175.73 ; 0.9,0.9))\end{array}$ \\
\hline & $\begin{array}{c}((51.9,94.05,125.4,177.75 ; 1,1) \\
(67.67,103.5,115.95,147.73 ; 0.9,0.9))\end{array}$ & $\begin{array}{c}((13,22.5,30,42.5 ; 1,1) \\
(16.8,24,28.5,36.8 ; 0.9,0.9))\end{array}$ & $\begin{array}{c}((51.9,94.05,125.4,177.75 ; 1,1) \\
(67.67,103.5,115.95,147.73 ; 0.9,0.9))\end{array}$ \\
\hline & $\begin{array}{c}((87,201.67,288,434.5 ; 1,1) \\
(136.8,224.67,257.83,358.8 ; 0.9,0.9))\end{array}$ & $\begin{array}{c}((62.1,123.63,168,231.35 ; 1,1) \\
(86.87,136.63,153.82,198.13 ; 0.9,0.9))\end{array}$ & $\begin{array}{c}((136.1,302.8,426,3623.35 ; 1,1) \\
(206.87,337.3,383.15,520.13 ; 0.9,0.9))\end{array}$ \\
\hline & $((136.1,302.8,426,623.35 ; 1,1)$ & $((51.9,94.05,125.4,177.75 ; 1,1)$ & $((136.1,302.8,426,3623.35 ; 1,1)$ \\
\hline & $(206.87,337.3,383.15,520.13 ; 0.9,0.9))$ & $(67.67,103.5,115.95,147.73 ; 0.9,0.9))$ & $(206.87,337.3,383.15,520.13 ; 0.9,0.9))$ \\
\hline & $\begin{array}{c}((88.35,221.33,314.3,482.3 ; 1,1) \\
(145.4,246.82,284.1,399 ; 0.9,0.9))\end{array}$ & $\begin{array}{c}((64.7,114.05,154.2,203.35 ; 1,1) \\
(84.47,125.9,141.55,175.73 ; 0.9,0.9))\end{array}$ & $\begin{array}{c}((136.1,302.8,426,3623.35 ; 1,1) \\
(206.87,337.3,383.15,520.13 ; 0.9,0.9))\end{array}$ \\
\hline
\end{tabular}

act.: Activities.

For example:

$$
\left.\begin{array}{rl}
\tilde{\tilde{E}}_{3}= & \max \left\{w_{2,2}(3), w_{3,1}(3)\right\} \\
= & \max \left\{\begin{array}{l}
((35,54.67,68.3,94 ; 1,1), \\
(44.3,61.5,61.5,80.67 ; 0.9,0.9)), \\
((51.9,94,125.4,177.75 ; 1,1), \\
(67.67,103.5,115.95,147.73 ; 0.90 .9))
\end{array}\right.
\end{array}\right\}
$$

In addition, IT2F-ES time and IT2F-EF time of each node $i$ are determined via Eqs. (21) and (22) and illustrated in Table 13.
For example:

$$
\begin{aligned}
E \tilde{\tilde{S}} T_{(3,5)}= & \tilde{\tilde{E}}_{3}=((51.9,94,125.4,177.75 ; 1,1) \\
& (67.67,103.5,115.95,147.73 ; 0.90 .9)) \\
E \tilde{\tilde{F}} T_{(3,5)}= & E \tilde{\tilde{S}} T_{(3,5)}+\tilde{\tilde{T}}_{(3,5)} \\
= & ((51.9,94,125.4,177.75 ; 1,1) \\
& (67.67,103.5,115.95,147.73 ; 0.90 .9)) \\
& \oplus((84.2,208.75,300.6,445.6 ; 1,1) \\
& (139.2,233.8,267.2,372.4 ; 0.9,0.9)) \\
= & ((136.1,302.8,426,623.35 ; 1,1),
\end{aligned}
$$

$(206.87,337.3,383.15,520.13 ; 0.9,0.9))$. 
Finally, the IT2F-latest start and finish times are obtained by means of Eqs. (23) and (24), respectively. The results are demonstrated in Table 13.

For example:

$$
\begin{aligned}
L \tilde{\tilde{S}} T_{(3,5)}= & T \tilde{\tilde{F}}_{(3,5)}+E \tilde{\tilde{S}} T_{(3,5)} \\
= & ((0,0,0,0 ; 1,1),(0,0,0,0 ; 0.9,0.9)) \\
& \oplus((51.9,94,125.4,177.75 ; 1,1), \\
& (67.67,103.5,115.95,147.73 ; 0.90 .9)) \\
= & ((51.9,94,125.4,177.75 ; 1,1),
\end{aligned}
$$

$(67.67,103.5,115.95,147.73 ; 0.90 .9))$,

and:

$$
\begin{aligned}
L \tilde{\tilde{F}} T_{(3,5)}= & L \tilde{\tilde{S}} T_{(3,5)}+\tilde{\tilde{T}}_{(3,5)} \\
= & ((51.9,94,125.4,177.75 ; 1,1), \\
& (67.67,103.5,115.95,147.73 ; 0.90 .9)) \\
& \oplus((84.2,208.75,300.6,445.6 ; 1,1), \\
& (139.2,233.8,267.2,372.4 ; 0.9,0.9)) \\
= & ((136.1,302.8,426,3623.35 ; 1,1),
\end{aligned}
$$

$(206.87,337.3,383.15,520.13 ; 0.9,0.9))$.

The criticality degree of each activity and critical path can be determined, as shown in Table 14. The IT2F$\mathrm{TF}$ of each activity is converted to a crisp number by using Eq. (A.8). It is obvious that the critical activities are $1-2,2-3$, and $3-5$; nevertheless, the critical path is the path $1-2-3-5$.

In order to show the advantages of the proposed method, a simple example is solved by two methods of the literature. If $\tilde{A}, \tilde{B}$ are defined as follows:

$$
\begin{gathered}
\tilde{A}=(10,15,30,40), \\
\tilde{B}=(12,13,15,20),
\end{gathered}
$$

then the subtraction operation is performed by the methodology of Kumar and Kaur [47] as follows:

$$
\tilde{A} \Theta \tilde{B}=(5,10,10,11) .
$$

Further, the subtraction operation is computed by the method of Rao and Shankar [48] as follows:

$$
\tilde{A} \Theta \tilde{B}=(0,0,17,28) .
$$

Finally, this operation is carried out by using the proposed method as follows:

$$
\tilde{A} \Theta \tilde{B}=(0,0,15,20) .
$$

The classic fuzzy subtraction operation is calculated as follows:

$$
\begin{aligned}
\tilde{A} \Theta \tilde{B} & =(10,15,30,40) \Theta(12,13,15,20) \\
& =(-10,0,17,28) \stackrel{\text { crisp number }}{\longrightarrow}=\frac{35}{4} .
\end{aligned}
$$

With this in mind, the crisp results of the proposed method, and methods of Kumar and Kaur [47], and Rao and Shankar [48] are calculated as follows:

Kumar and Kaur [47] method:

$$
\left(\frac{5+10+10+11}{4}\right)=\frac{36}{4},
$$

Rao and Shankar [48] method:

$$
\left(\frac{0+0+17+28}{4}\right)=\frac{45}{4},
$$

Proposed method:

Table 14. Criticality degree of each activity.

\begin{tabular}{clc}
\hline Activities & \multicolumn{1}{c}{ Total float of each activity } & Criticality degree \\
\hline $1-2$ & $((0,0,0,0 ; 1,1),(0,0,0,0 ; 0.9,0.9))$ & 0 \\
$1-3$ & $((16.9,39.38,57.06,83.75 ; 1,1),(23.3,42,54.45,67.07 ; 0.9,0.9))$ & 47.95 \\
$1-4$ & $((47.75,81.47,111.7,141.07 ; 1,1),(61.4,90.48,99.05,121.13 ; 0.9,0.9))$ & 94.21 \\
$2-3$ & $((0,0,0,0 ; 1,1),(0,0,0,0 ; 0.9,0.9))$ & 0 \\
$2-5$ & $((49.1,101.13,138,188.85 ; 1,1),(70.07,112.63,125.32,161.3 ; 0.9,0.9))$ & 118.26 \\
$3-5$ & $((0,0,0,0 ; 1,1),(0,0,0,0 ; 0.9,0.9))$ & 0 \\
$4-5$ & $((47.75,81.47,111.7,141.07 ; 1,1),(61.4,90.48,99.05,121.13 ; 0.9,0.9))$ & 94.21 \\
\hline
\end{tabular}




$$
\left(\frac{0+0+15+20}{4}\right)=\frac{35}{4} .
$$

As shown above, the previous methods compute the amounts larger than the actual amount. However, the results of classic fuzzy subtraction and the proposed method are the same. In fact, the proposed method produces real amounts in subtraction operation and avoids negative numbers.

\section{Comparative analysis}

In this section, to validate the results of characteristics of each task and critical path, a new proposed approach is applied to the two examples from recent literature. Note that these examples are solved by the proposed method under traditional fuzzy environment. The first example is related to Sireesha and Shankar [51] and is solved in the triangle fuzzy environment, and the second example is relevant to Sireesha and Shankar [52] and is solved in the trapezoidal fuzzy environment. These two examples are depicted in Tables 15 and 16, respectively. In these two cases, it is observed that the criticality degree is the same, showing the validity of the proposed method. It is obvious that the proposed method avoids producing negative number in determining the characteristics of each activity.

The proposed method has been compared with two methods explored in the literature, and the results confirmed the viability of proposed method. However, these two methods of the literature have produced negative number in determining the characteristics of the fuzzy project network. As seen in Tables 13 and 14, the proposed method avoided producing negative number in determining the characteristics of fuzzy project network, and the results were the same. Nevertheless, the validity of the proposed method was confirmed based on Tables 13 and 14 .

\section{Conclusions}

In this paper, IT2FSs were used to consider the risks of each activity and determine the critical path of the project network. A new method for determining the correlation among risk factors was also extended under the IT2F environment to tackle the uncertainty of real-world projects better. Then, a new subtraction operator was introduced to avoid producing negative numbers in the project network, and the

Table 15. Comparative analysis of the proposed method and the method of Sireesha and Shankar [51] in the case of the first example.

\begin{tabular}{ccccc}
\hline Activities & $\begin{array}{c}\text { Sireesha and Shankar [51] } \\
\boldsymbol{T} \tilde{\boldsymbol{F}}_{\boldsymbol{i} \boldsymbol{j}}\end{array}$ & $\begin{array}{c}\text { Criticality } \\
\text { degree }\end{array}$ & $\begin{array}{c}\text { Proposed } \\
\text { method }\end{array}$ & $\begin{array}{c}\text { Criticality } \\
\text { degree }\end{array}$ \\
\hline $1-2$ & $(-7,0,6,14)$ & 3.25 & $(2,3,3,5)$ & 3.25 \\
$1-3$ & $(-12,-3,3,12)$ & 0 & $(0,0,0,0)$ & 0 \\
$1-5$ & $(2,6,10,18)$ & 9 & $(6,7,9,14)$ & 9 \\
$2-4$ & $(-7,0,6,14)$ & 3.25 & $(2,3,3,5)$ & 3.25 \\
$2-5$ & $(-5,2,7,16)$ & 5 & $(4,4,5,7)$ & 5 \\
$3-4$ & $(-6,2,6,15)$ & 4.25 & $(3,3,5,6)$ & 4.25 \\
$3-6$ & $(-12,-3,3,12)$ & 0 & $(0,0,0,0)$ & 0 \\
$4-5$ & $(-7,0,6,14)$ & 3.25 & $(2,3,3,5)$ & 3.25 \\
$4-6$ & $(-6,2,6,15)$ & 3.75 & $(2,3,4,6)$ & 3.75 \\
$5-6$ & $(-7,0,6,14)$ & 3.25 & $(2,3,3,5)$ & 3.25 \\
\hline
\end{tabular}

Table 16. Comparative analysis of the proposed method and the method of Sireesha and Shankar [52] in the case of the second example.

\begin{tabular}{ccccc}
\hline Activities & $\begin{array}{c}\text { Sireesha and Shankar [52] } \\
\boldsymbol{T} \tilde{\boldsymbol{F}}_{\boldsymbol{i}}\end{array}$ & $\begin{array}{c}\text { Criticality } \\
\text { degree }\end{array}$ & $\begin{array}{c}\text { Proposed } \\
\text { method }\end{array}$ & $\begin{array}{c}\text { Criticality } \\
\text { degree }\end{array}$ \\
\hline $1-2$ & $(-160,0,160)$ & 0 & $(0,0,0)$ & 0 \\
$1-3$ & $(-130,20,170)$ & 20 & $(10,20,30)$ & 20 \\
$1-4$ & $(-110,37,185)$ & 37.3 & $(25,37,50)$ & 37.3 \\
$2-3$ & $(-160,0,160)$ & 0 & $(0,0,0)$ & 0 \\
$2-5$ & $(-100,45,190)$ & 45 & $(30,45,60)$ & 45 \\
$3-5$ & $(-160,0,160)$ & 0 & $(0,0,0)$ & 0 \\
$4-5$ & $(-110,37,185)$ & 37.3 & $(25,37,50)$ & 37.3 \\
\hline
\end{tabular}


NWRT method was modified by this new operator. Furthermore, the NWRT method was developed under IT2FSs. An example from literature was solved to demonstrate the calculation procedure and capability of the proposed method. For the comparative analysis, the total float of each activity of two examples from the recent literature was obtained; finally, the results were defuzzified to determine the criticality degree. It was observed that the criticality degree of the two previous decision methods was the same as the results of the proposed method. The main advantage of the proposed method was the production of positive numbers, because the negative numbers have no physical meanings in a project network. The new subtraction operator for critical path computation did not generate larger values than real values in comparison with previous methods. In fact, the new subtraction operator enjoyed two advantages. Firstly, it avoided producing negative number; secondly, it avoided producing lager values than real values. Moreover, the presented approach was simpler and easier than traditional CPM calculation, and the critical path would be determined directly based on the rooted tree. A mathematical modeling approach of the problem can be set for the future research. The proposed methodology will require more inputs from the DMs, which can be time consuming. To solve this problem, a new operator for considering the correlation between risk factors and obtaining probability can be added to the proposed method. Furthermore, the proposed method can be extended under the group decision environment to achieve a higher degree of accuracy.

\section{Acknowledgements}

The authors thank anonymous referees for valuable comments, which improved the primary version of this paper.

\section{References}

1. Kerzner, H., Project Management: A Systems Approach to Planning, Scheduling, and Controlling, 12th Edn., John Wiley \& Sons, US (2017).

2. Malcolm, D.G., Roseboom, J.H., Clark, C.E., and Fazar, W. "Application of a technique for research and development program evaluation", Operations Research, 7(5), pp. 646-669 (1959).

3. Chanas, S. and Zieliński, P. "Critical path analysis in the network with fuzzy activity times", Fuzzy Sets and Systems, 122(2), pp. 195-204 (2001).

4. Zareei, A., Zaerpour, F., Bagherpour, M., Noora, A.A., and Vencheh, A.H. "A new approach for solving fuzzy critical path problem using analysis of events", Expert Systems with Applications, 38(1), pp. 87-93 (2011).
5. Zadeh, L.A. "Fuzzy sets as basis for a theory of possibility", Fuzzy Sets and Systems, 1, pp. 3-28 (1978).

6. Project Management Institute, A Guide to the Project Management Body of Knowledge (PM-BOK Guide), 5th Edn., US (2013).

7. Espinoza, R.D. "Separating project risk from the time value of money: A step toward integration of risk management and valuation of infrastructure investments", International Journal of Project Management, 32(6), pp. 1056-1072 (2014).

8. Yim, R., Castaneda, J., Doolen, T., Tumer, I., and Malak, R. "A study of the impact of project classification on project risk indicators", International Journal of Project Management, 33(4), pp. 863-876 (2015).

9. Golini, R., Kalchschmidt, M., and Landoni, P. "Adoption of project management practices: The impact on international development projects of nongovernmental organizations", International Journal of Project Management, 33(3), pp. 650-663 (2015).

10. Carvalho, M.M.D. and Rabechini Junior, R. "Impact of risk management on project performance: The importance of soft skills", International Journal of Production Research, 53(2), pp. 321-340 (2015).

11. Muriana, C. and Vizzini, G. "Project risk management: A deterministic quantitative technique for assessment and mitigation", International Journal of Project Management, 35(3), pp. 320-340 (2017).

12. Kuchta, D. "Use of fuzzy numbers in project risk (criticality) assessment", International Journal of Project Management, 19(5), pp. 305-310 (2001).

13. Smith, N.J., Appraisal, Risk and Uncertainty, 1st Ed., Thomas Telford UK (2003).

14. Książek, M.V., Nowak, P.O., Kivrak, S., Rosłon, J.H., and Ustinovichius, L. "Computer-aided decisionmaking in construction project development", Journal of Civil Engineering and Management, 21(2), pp. 248259 (2015).

15. Barber, R.B. "Understanding internally generated risks in projects", International Journal of Project Management, 23(8), pp. 584-590 (2005).

16. Ebrahimnejad, S., Mousavi, S.M., TavakkoliMoghaddam, R., and Heydar, M. "Evaluating high risks in large-scale projects using an extended VIKOR method under a fuzzy environment", International Journal of Industrial Engineering Computations, 3, pp. 463-476 (2012).

17. Mousavi, S.M., Tavakkoli-Moghaddam, R., Azaron, A., Mojtahedi, S.M.H., and Hashemi, H. "Risk assessment for highway projects using Jackknife technique", Expert Systems with Applications, 38, pp. 5514-5524 (2011).

18. Mousavi, S.M., Tavakkoli-Moghaddam, R., Hashemi, H., and Mojtahedi, S.M.H. "A novel approach based on non-parametric resampling with the interval analysis for large engineering project risks", Safety Science, 49, pp. $1340-1348$ (2011). 
19. Hashemi, H., Mousavi, S.M., and Mojtahedi, S.M.H. "Bootstrap technique for risk analysis with interval numbers in bridge construction projects", Journal of Construction Engineering and Management - ASCE, 137(8), pp. 600-608 (2011).

20. Makui, A., Mojtahedi, S.M.H., and Mousavi, S.M. "Project risk identification and analysis based on group decision making methodology in a fuzzy environment", International Journal of Management Science and Engineering Management, 5(2), pp. 108-118 (2010).

21. El-Sayegh, S.M. "Risk assessment and allocation in the UAE construction industry", International Journal of Project Management, 26(4), pp. 431-438 (2008).

22. Liang, G.S. and Han, T.C. "Fuzzy critical path for project network", Information and Management Sciences, 15(4), pp. 29-40 (2004).

23. Kumar, A. and Kaur, P. "A new method for fuzzy critical path analysis in project networks with a new representation of triangular fuzzy numbers", Applications and Applied Mathematics: An International Journal, 5(10), pp. 1442-1466 (2010).

24. Shankar, N.R. and Saradhi, B.P. "Fuzzy critical path method in interval-valued activity networks", International Journal of Pure and Applied Sciences and Technology, 3(2), pp. 72-79 (2011).

25. Taylan, O., Bafail, A.O., Abdulaal, R.M., and Kabli, M.R. "Construction projects selection and risk assessment by fuzzy AHP and fuzzy TOPSIS methodologies", Applied Soft Computing, 17, pp. 105-116 (2014).

26. Kuchta, D. and Ptaszyńska, E. "Fuzzy based risk register for construction project risk assessment", AIP Conference Proceedings, 1863(1), pp. 1011-1019 (2017).

27. Asan, U.M.U.T., Soyer, A., and Bozdag, E. "An interval type-2 fuzzy prioritization approach to project risk assessment", Journal of Multiple-Valued Logic and Soft Computing, 26(6), pp. 541-577 (2016).

28. Mendel, J. and Wu, D., Perceptual Computing: Aiding People in Making Subjective Judgments, 1st Ed., John Wiley \& Sons, US (2010).

29. Mendel, J.M. "Type-2 fuzzy sets and systems: An overview", IEEE Computational Intelligence Magazine, 2(2), pp. 20-29 (2007).

30. Deveci, M., Demirel, N.Ç., and Ahmetoğlu, E. "Airline new route selection based on interval type- 2 fuzzy MCDM: A case study of new route between TurkeyNorth American region destinations", Journal of Air Transport Management, 59, pp. 83-99 (2017).

31. Qin, J., Liu, X., and Pedrycz, W. "An extended TODIM multi-criteria group decision making method for green supplier selection in interval type-2 fuzzy environment", European Journal of Operational Research, 258(2), pp. 626-638 (2017).

32. Abdullah, L. and Najib, L. "A new type-2 fuzzy set of linguistic variables for the fuzzy analytic hierarchy process", Expert Systems with Applications, 41(7), pp. 3297-3305 (2014).
33. Ghorabaee, M.K., Amiri, M., Zavadskas, E.K., Turskis, Z., and Antucheviciene, J. "A new multicriteria model based on interval type-2 fuzzy sets and EDAS method for supplier evaluation and order allocation with environmental considerations", Computers \& Industrial Engineering, 112, pp. 156-174 (2017).

34. Abdullah, L. and Zulkifli, N. "A new DEMATEL method based on interval type-2 fuzzy sets for developing causal relationship of knowledge management criteria", Neural Computing and Applications, 29, pp. 1-17 (2018).

35. Kilic, M. and Kaya, I. "Investment project evaluation by a decision making methodology based on type-2 fuzzy sets", Applied Soft Computing, 27, pp. 399-410 (2015).

36. Ghorabaee, M.K., Amiri, M., Sadaghiani, J.S., and Zavadskas, E.K. "Multi-criteria project selection using an extended VIKOR method with interval type-2 fuzzy sets", International Journal of Information Technology \& Decision Making, 14(5), pp. 993-1016 (2015).

37. Oztaysi, B. "A group decision making approach using interval type-2 fuzzy AHP for enterprise information systems project selection", Journal of Multiple-Valued Logic \& Soft Computing, 24(5), pp. 475-500 (2015).

38. Bozdag, E., Asan, U., Soyer, A., and Serdarasan, S. "Risk prioritization in failure mode and effects analysis using interval type-2 fuzzy sets", Expert Systems with Applications, 42(8), pp. 4000-4015 (2015).

39. Mohagheghi, V., Mousavi, S.M., and Vahdani, B. "Analyzing project cash flow by a new interval type- 2 fuzzy model with an application to construction industry", Neural Computing and Applications, 28(11), pp. 33933411 (2017).

40. Mohagheghi, V., Mousavi, S.M., Vahdani, B., and Shahriari, M.R. "R\&D project evaluation and project portfolio selection by a new interval type- 2 fuzzy optimization approach", Neural Computing and Applications, 28(12), pp. 3869-3888 (2017).

41. Zadeh, L.A. "Fuzzy sets", Information and Control, 8(3), pp. 338-353 (1965).

42. Zadeh, L.A. "The concept of a linguistic variable and its application to approximate reasoning-I", Information Sciences, 8(3), pp. 199-249 (1975).

43. Chen, T.Y. "A linear assignment method for multiplecriteria decision analysis with interval type-2 fuzzy sets", Applied Soft Computing, 13(5), pp. 2735-2748 (2013).

44. Bezdek, J.C., Pattern Recognition with Fuzzy Objective Function Algorithms, 1st Edn., Springer Science \& Business Media, US (2013).

45. Celikyilmaz, A. and Turksen, I.B. "Modeling uncertainty with fuzzy logic", Studies in Fuzziness and Soft Computing, 240, pp. 149-215 (2009).

46. Shankar, N.R., Sireesha, V., and Rao, P.P.B. "An analytical method for finding critical path in a fuzzy project network", International Journal of Contemporary Mathematical Sciences, 5(20), pp. 953-962 (2010). 
47. Kumar, A. and Kaur, P. "A new approach for fuzzy critical path analysis", International Journal of Mathematics in Operational Research, 3(3), pp. 341-357 (2011).

48. Rao, P.P.B. and Shankar, N.R. "Fuzzy critical path analysis based on centroid of centroids of fuzzy numbers and new subtraction method", International Journal of Mathematics in Operational Research, 5(2), pp. 205-224 (2013).

49. Yakhchali, S.H. and Ghodsypour, S.H. "Computing latest starting times of activities in interval-valued networks with minimal time lags", European Journal of Operational Research, 200(3), pp. 874-880 (2010).

50. Yakhchali, S.H. and Ghodsypour, S.H. "On the latest starting times and criticality of activities in a network with imprecise durations", Applied Mathematical Modelling, 34(8), pp. 2044-2058 (2010).

51. Sireesha, V. and Shankar, N.R. "A new approach to find project characteristics and multiple possible critical paths in a fuzzy project network", Fuzzy Information and Engineering, 5(1), pp. 69-85 (2013).

52. Sireesha, V. and Shankar, N.R. "A node-weighted rooted tree (NWRT) method to find project characteristics and critical path in a triangular fuzzy project network", Computational and Applied Mathematics, 37(2), pp. 1971-1985 (2018).

53. Madhuri, K.U., Babu, S.S., and Shankar, N.R. "Fuzzy risk analysis based on the novel fuzzy ranking with new arithmetic operations of linguistic fuzzy numbers", Journal of Intelligent \& Fuzzy Systems, 26(5), pp. 2391-2401 (2014).

54. Hu, J., Zhang, Y., Chen, X., and Liu, Y. "Multicriteria decision making method based on possibility degree of interval type-2 fuzzy number", KnowledgeBased Systems, 43, pp. 21-29 (2013).

55. Mendel, J.M., John, R.I., and Liu, F. "Interval type-2 fuzzy logic systems made simple", IEEE Transactions on Fuzzy Systems, 14(6), pp. 808-821 (2006).

56. Chen, S.M. and Lee, L.W. "Fuzzy multiple attributes group decision-making based on the interval type-2 TOPSIS method", Expert Systems with Applications, 37(4), pp. 2790-2798 (2010).

57. Hu, J., Xiao, K., Chen, X., and Liu, Y. "Interval type-2 hesitant fuzzy set and its application in multicriteria decision making", Computers \& Industrial Engineering, 87, pp. 91-103 (2015).

58. Li, D. and Gu, Y. "Method for ranking interval numbers based on possibility degree", Xitong Gongcheng Xuebao, 23(2), pp. 243-261 (2008).

\section{Appendix}

The membership degree of the T1FS is a crisp number in $[0,1]$. Usually, there are circumstances where it is difficult to specify the exact membership function for a fuzzy set. It is not appropriate to use T1FSs in this situation. To overcome this issue, Zadeh [42] presented T2FSs, which are the extended form of T1FSs. T2FSs are defined by both primary and secondary memberships to achieve higher degrees of freedom and flexibility, and they are three-dimensional. Hence, T2FSs have the advantage of modeling uncertainty more accurately than T1FSs [54]. T2FSs are more appropriate, flexible and intelligent than T1FSs in illustrating uncertainties for handling fuzzy group decision problems [55-57]. Moreover, the computational burden is significant when using T2FSs to solve problems [58]. IT2FSs can be considered as a special case of general T2FS, where all the amounts of secondary membership are equal to 1 [55]. Therefore, it not only demonstrates uncertainty better than T1FSs, but also reduces the computation burden when compared with T2FSs.

A T2FS $\tilde{\tilde{A}}$ in the universe of discourse $\mathrm{X}$ can be illustrated by a Type- 2 membership function $\mu \tilde{\tilde{A}}$ demonstrated below [55]:

$$
\begin{aligned}
\tilde{\tilde{A}}= & \left\{\left((x, u), \mu_{\tilde{\tilde{A}}}(x, u)\right) \mid \forall x \in X, \forall u \in J_{x}\right. \\
& \left.\subseteq[0,1], 0 \leq \mu_{\tilde{\tilde{A}}}(x, u) \leq 1\right\},
\end{aligned}
$$

where $J_{x}$ demonstrates an interval in $[0,1]$. However, the T2FS $\tilde{\tilde{A}}$ can also be shown by using the following:

$$
\tilde{\tilde{A}}=\int_{x \in X} \int_{u \in J_{x}} \mu_{\tilde{\tilde{A}}}(x, u) /(x, u),
$$

where $J_{x} \subseteq[0,1]$ and $\iint$ demonstrate union over all admissible $x$ and $u$.

If all $\mu_{\tilde{A}}(x, u)=1$, then $\tilde{\tilde{A}}$ is called IT2FS. An IT2FS $\tilde{\tilde{A}}$ can be viewed as a special case of T2FSs as demonstrated below:

$$
\tilde{\tilde{A}}=\int_{x \in X} \int_{u \in J_{x}} 1 /(x, u) .
$$

The upper membership grade and the lower membership grade of an IT2FS are Type-1 membership grades, respectively. Figure A.1 illustrates a trapezoidal IT2F:

$$
\begin{aligned}
\tilde{\tilde{A}}_{i}= & \left(\tilde{A}_{i}^{U}, \tilde{A}_{i}^{L}\right) \\
= & \left(\left(a_{i 1}^{U}, a_{i 2}^{U}, a_{i 3}^{U}, a_{i 4}^{U} ; H_{1}\left(\tilde{A}_{i}^{U}\right), H_{1}\left(\tilde{A}_{i}^{U}\right)\right),\right. \\
& \left.\left(a_{i 1}^{L}, a_{i 2}^{L}, a_{i 3}^{L}, a_{i 4}^{L} ; H_{1}\left(\tilde{A}_{i}^{L}\right), H_{1}\left(\tilde{A}_{i}^{L}\right)\right)\right),
\end{aligned}
$$

where $\tilde{A}_{i}^{U}$ and $\tilde{A}_{i}^{L}$ are T1FSs; $a_{i 1}^{U}, a_{i 2}^{U}, a_{i 3}^{U}, a_{i 4}^{U}$, $a_{i 1}^{L}, \quad a_{i 2}^{L}, \quad a_{i 3}^{L}$ and $a_{i 4}^{L}$ are the reference points of the IT2F $\tilde{A}_{i} ; H_{j}\left(\tilde{A}_{i}^{U}\right)$ denotes the membership value of the 


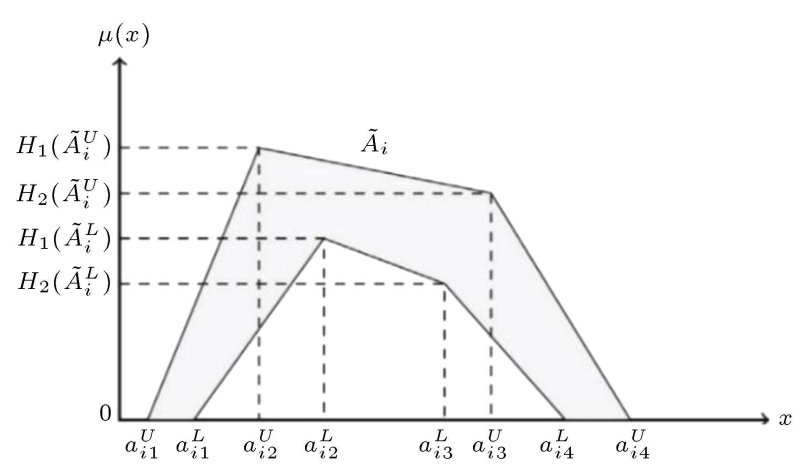

Figure A.1. Trapezoidal interval Type-2 fuzzy set.

element $a_{i(j+1)}^{U}$ in the upper trapezoidal membership function $\tilde{A}_{i}^{U}, 1 \leq j \leq 2$; and $H_{j}\left(\tilde{A}_{i}^{L}\right)$ denotes the membership value of the element $a_{i(j+1)}^{L}$ in the lower trapezoidal membership function $\tilde{A}_{i}^{L}, 1 \leq j \leq 2$ [55].

The basic algebraic operations between trapezoidal IT2FSs are described as follows [56]:

$$
\begin{aligned}
\tilde{\tilde{F}}_{1}= & \left(\tilde{F}_{1}^{U}, \tilde{F}_{1}^{L}\right) \\
= & \left(\left(f_{11}^{U}, f_{12}^{U}, f_{13}^{U}, f_{14}^{U} ; H_{1}\left(\tilde{F}_{1}^{U}\right), H_{2}\left(\tilde{F}_{1}^{U}\right)\right),\right. \\
& \left.\left(f_{11}^{L}, f_{12}^{L}, f_{13}^{L}, f_{14}^{L} ; H_{1}\left(\tilde{F}_{1}^{L}\right), H_{2}\left(\tilde{F}_{1}^{L}\right)\right)\right), \\
\tilde{\tilde{F}}_{2}= & \left(\tilde{F}_{2}^{U}, \tilde{F}_{2}^{L}\right) \\
= & \left(\left(f_{21}^{U}, f_{22}^{U}, f_{23}^{U}, f_{24}^{U} ; H_{1}\left(\tilde{F}_{2}^{U}\right), H_{2}\left(\tilde{F}_{2}^{U}\right)\right),\right. \\
& \left.\left(f_{21}^{L}, f_{22}^{L}, f_{23}^{L}, f_{24}^{L} ; H_{1}\left(\tilde{F}_{2}^{L}\right), H_{2}\left(\tilde{F}_{2}^{L}\right)\right)\right)
\end{aligned}
$$

The addition operation:

$$
\begin{aligned}
\tilde{\tilde{F}}_{1} \oplus \tilde{\tilde{F}}_{2}=\left(\tilde{F}_{1}^{U}, \tilde{F}_{1}^{L}\right)+\left(\tilde{F}_{2}^{U}, \tilde{F}_{2}^{L}\right) \\
=\left(\left(f_{11}^{U}+f_{21}^{U}, f_{12}^{U}+f_{22}^{U}, f_{13}^{U}+f_{23}^{U}, f_{14}^{U}+f_{24}^{U} ;\right.\right. \\
\quad \min \left(H_{1}\left(\tilde{F}_{1}^{U}\right), H_{1}\left(\tilde{F}_{2}^{U}\right)\right) \\
\left.\quad \min \left(H_{2}\left(\tilde{F}_{1}^{U}\right), H_{2}\left(\tilde{F}_{2}^{U}\right)\right)\right) \\
\quad f_{11}^{L}+f_{21}^{L}, f_{12}^{L}+f_{22}^{L}, f_{13}^{L}+f_{23}^{L}, f_{14}^{L}+f_{24}^{L} ; \\
\quad \min \left(H_{1}\left(\tilde{F}_{1}^{L}\right), H_{1}\left(\tilde{F}_{2}^{L}\right)\right) \\
\left.\left.\quad \min \left(H_{2}\left(\tilde{F}_{1}^{L}\right), H_{2}\left(\tilde{F}_{2}^{L}\right)\right)\right)\right) .
\end{aligned}
$$

The subtraction operation:

$$
\begin{aligned}
\tilde{\tilde{F}}_{1} \Theta \tilde{\tilde{F}}_{2}=\left(\tilde{F}_{1}^{U}, \tilde{F}_{1}^{L}\right)-\left(\tilde{F}_{2}^{U}, \tilde{F}_{2}^{L}\right) \\
=\left(\left(f_{11}^{U}-f_{24}^{U}, f_{12}^{U}-f_{23}^{U}, f_{13}^{U}-f_{22}^{U}, f_{14}^{U}-f_{21}^{U} ;\right.\right. \\
\quad \min \left(H_{1}\left(\tilde{F}_{1}^{U}\right), H_{1}\left(\tilde{F}_{2}^{U}\right)\right) \\
\left.\quad \min \left(H_{2}\left(\tilde{A}_{1}^{U}\right), H_{2}\left(\tilde{A}_{2}^{U}\right)\right)\right) \\
\quad f_{11}^{L}-f_{24}^{L}, f_{12}^{L}-f_{23}^{L}, f_{13}^{L}-f_{22}^{L}, f_{14}^{L}-f_{21}^{L} ; \\
\quad \min \left(H_{1}\left(\tilde{F}_{1}^{L}\right), H_{1}\left(\tilde{F}_{2}^{L}\right)\right) \\
\left.\left.\quad \min \left(H_{2}\left(\tilde{F}_{1}^{L}\right), H_{2}\left(\tilde{F}_{2}^{L}\right)\right)\right)\right) .
\end{aligned}
$$

The multiplication operation:

$$
\begin{aligned}
\tilde{\tilde{F}}_{1} \otimes \tilde{\tilde{F}}_{2}=\left(\tilde{F}_{1}^{U}, \tilde{F}_{1}^{L}\right) \times\left(\tilde{F}_{2}^{U}, \tilde{F}_{2}^{L}\right) \\
=\left(\left(f_{11}^{U} \times f_{21}^{U}, f_{12}^{U} \times f_{22}^{U}, f_{13}^{U} \times f_{23}^{U}, f_{14}^{U} \times f_{24}^{U} ;\right.\right. \\
\quad \min \left(H_{1}\left(\tilde{F}_{1}^{U}\right), H_{1}\left(\tilde{F}_{2}^{U}\right)\right), \\
\left.\quad \min \left(H_{2}\left(\tilde{F}_{1}^{U}\right), H_{2}\left(\tilde{F}_{2}^{U}\right)\right)\right), \\
\quad f_{11}^{L} \times f_{21}^{L}, f_{12}^{L} \times f_{22}^{L}, f_{13}^{L} \times f_{23}^{L}, f_{14}^{L} \times f_{24}^{L} ; \\
\quad \min \left(H_{1}\left(\tilde{F}_{1}^{L}\right), H_{1}\left(\tilde{F}_{2}^{L}\right)\right), \\
\left.\left.\quad \min \left(H_{2}\left(\tilde{F}_{1}^{L}\right), H_{2}\left(\tilde{F}_{2}^{L}\right)\right)\right)\right) .
\end{aligned}
$$

The defuzzified value of a trapezoidal IT2FN is defined as follows [36]:

$$
\begin{gathered}
\operatorname{Def}\left(\tilde{\tilde{F}}_{1}\right)=\frac{1}{2}\left(\sum_{T \in\{U, L\}}\right. \\
\left.\frac{f_{11}^{T}+H_{1}\left(\tilde{F}_{1}^{T}\right) f_{12}^{T}+H_{2}\left(\tilde{F}_{1}^{T}\right) f_{13}^{T}+f_{14}^{T}}{2+H_{1}\left(\tilde{F}_{1}^{T}\right)+H_{2}\left(\tilde{F}_{1}^{T}\right)}\right) .
\end{gathered}
$$

\section{Biographies}

Yahya Dorfeshan received an MSc degree from the Department of Industrial Engineering, Faculty of Engineering, Shahed University, Tehran, Iran. His main research interests include quantitative methods in project management, fuzzy sets theory, multicriteria decision-making under uncertainty, and applied operations research. He has published several 
papers in international journals and conference proceedings.

Seyed Meysam Mousavi is an Associate Professor at the Department of Industrial Engineering, Faculty of Engineering, Shahed University in Tehran, Iran. He received a $\mathrm{PhD}$ degree from the School of Industrial Engineering at University of Tehran, Iran, and is currently a member of Iran's National Elite Foundation. He is the Head of Industrial Engineering Department at Shahed University and a member of the Iranian Operational Research Association. His main research interests include cross-docking systems planning, logistics planning and scheduling, quantitative methods in project management, engineering optimization under uncertainty, multiple criteria decision making under uncertainty, and applied soft computing. He has published many papers and book chapters in reputable journals and international conference proceedings.
Behnam Vahdani is an Assistant Professor at Faculty of Industrial and Mechanical Engineering, Qazvin Branch, Islamic Azad University in Iran and is a member of Iran's National Elite Foundation. His current research interests include supply chain network design, facility location and design, multi-criteria decision making, uncertain programming, meta-heuristics algorithms, and operations research applications. He has published numerous papers and book chapters in the aforementioned areas.

Ali Siadat is a Professor at Laboratoire de Conception, Fabrication Commande, Arts et Métier Paris Tech, Centre de Metz in Metz, France. His current research interests include computer-aided manufacturing, modeling and optimization of manufacturing processes, decision-making in manufacturing, inspection planning, and operations research applications. He has published numerous papers and book chapters in the aforementioned fields. 